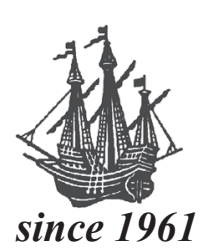

$\overline{\text { Baltica }}$

BALTICA Volume 34 Number 2 December 2021: 185-202

https://doi.org/10.5200/baltica.2021.2.5

\title{
Metamorphism of the Dizi Series Rocks (the Greater Caucasus): Petrography, Mineralogy and Evolution of Metamorphic Assemblages
}

\author{
Irakli Javakhishvili", David Shengelia, Leonid Shumlyanskyy, Tamara Tsutsunava, \\ Giorgi Chichinadze, Giorgi Beridze
}

Javakhishvili, I., Shengelia, D., Shumlyanskyy, L., Tsutsunava, T., Chichinadze, G., Beridze, G. 2021. Metamorphism of the Dizi Series Rocks (the Greater Caucasus): Petrography, Mineralogy and Evolution of Metamorphic Assemblages. Baltica, 34 (2), 185-202. Vilnius. ISSN 1648-858X.

Manuscript submitted 20 July 2021 / Accepted 26 October 2021 / Available online 20 December 2021

(C) Baltica 2021

Abstract. The Dizi Series is exposed within the Southern Slope zone of the Greater Caucasus, in the core of
the Svaneti anticlinorium. It is mainly composed of terrigenous, volcanogenic and carbonate rocks faunisti-
cally dated from the Devonian to the Triassic inclusive. Regional and contact metamorphism of the Dizi Series
rocks was studied. It is stated that the degree of regional metamorphism corresponds to the chlorite-sericite
subfacies of the greenschist facies, occurring at a temperature of $300-350^{\circ} \mathrm{C}$ and a pressure of $1.5-2.3 \mathrm{kbar}$.
As a result of the action of the Middle Jurassic intrusive rock bodies, the regionally metamorphosed rocks of
the Dizi Series underwent contact metamorphism. Three zones of contact metamorphism were distinguished
corresponding to albite-epidote-hornfels, andalusite-biotite-muscovite-chlorite-hornfels and andalusite-bio-
tite-muscovite-hornfels subfacies. Contact metamorphism took place at a significantly higher temperature and
lower pressure than the preceding regional metamorphism. The maximum temperature of the contact meta-
morphism reached $\approx 570^{\circ} \mathrm{C}$, while pressure varied within the range of $\approx 0.3-0.8$ kbar. The evolution of rock
associations of regional and contact metamorphism of the Dizi Series was studied. The fields of facies and
subfacies of regional and contact metamorphism are shown in the Ps-T diagram. Three age populations of zir-
cons were identified using U-Pb LA-ICP-MS dating of the diorite-porphyrite intrusion in the Dizi Series: Zrn1
(ca. $2200 \mathrm{Ma})$ and Zrn $2(458 \pm 29 \mathrm{Ma})$ that were captured by the diorite-porphyrite magma from the ancient
magmatic and metamorphic rocks of the crystalline basement, and Zrn3 (166.5 $\pm 4.6 \mathrm{Ma})$ that corresponds to
the age of diorite-porphyrite crystallization.

Keywords: low-temperature regional metamorphism; contact metamorphism zones; Ps-T conditions of metamorphism; magmatism; $\mathrm{U}-\mathrm{Pb}$ zircon dating

Irakli Javakhishvili* (irakli.javakhishvili@tsu.ge), David Shengelia (d.shenge@yahoo.com), Tamara Tsutsunava (tsutsunava@yahoo.com), Giorgi Chichinadze (giorgi.chichinadzegeolinst@gmail.com), Giorgi Beridze (giorgi.beridzegeolinst@gmail.com), Al. Janelidze Institute of Geology of Iv. Javakhishvili Tbilisi State University, 0186, Politkovskaya Str., 31, Tbilisi, Georgia; Leonid Shumlyanskyy(leonid.shumlyanskyy@curtin.edu.au), School of Earth and Planetary Sciences, Curtin University, GPO Box U1987, Perth, WA 6845, Australia

*Corresponding author

\section{INTRODUCTION}

The Dizi Series is exposed within the Southern Slope zone of the Greater Caucasus. The Caucasus represents a complex geological structure, which is a component of the Mediterranean (Alpine-Himalayan) collisional orogenic belt. Geological and paleo- magnetic data indicate that to the north of the ProtoPaleo-Tethys, a relatively small oceanic basin of the Southern slope of the Greater Caucasus was formed, apparently, already in the Late Precambrian. This basin was closed in the Bretonian phase (the DevonianCarboniferous boundary) of the Variscian orogeny (Gamkrelidze, Shengelia 2005). The Dizi Series was 
accumulated on the passive margin of the basin in conditions of the continental slope and its foot (Gamkrelidze, Shengelia 2005). The Dizi Series is characterized by a complex geological structure, evolution and conditions of metamorphism.

Although the stratigraphy and tectonics of the Dizi Series are well-studied, its metamorphism-related issues, in contrast to those of the rest of the pre-Alpine formations of the Greater Caucasus, are still insufficiently studied. Previous researchers (Goishvili 1960, 1961; Somin, Belov 1967; Adamia 1968; Somin 1971; Kutelia 1983; Somin 2011) have noted that the rocks of the series underwent regional and contact metamorphism. There is also an opinion that part of the Dizi Series has experienced anchimetamorphism (Gamkrelidze, Shengelia 2005; Chikhradze 2005). However, no detailed petrologic, mineralogic and geochronological studies have been carried out. It should be mentioned that previously there have been no analogues of the Dizi Series rocks established either in the Greater Caucasus or in the neighboring regions. Based on tectonic setting, lithology and age, it has been assumed recently that the Tauric flysch in the Crimea and the Dizi Series in the Caucasus represent parts of the same basin and were, most probably, deposited in the same fore-arc on the southern margin of Laurasia (Okay et al. 2015; Okay, Topuz 2016). Based on the conducted studies, the authors aimed to define the PT conditions of rock formation, specify the relationship between regional and contact metamorphism in the Dizi Series, and clarify some aspects of the geodynamic evolution of the region.

\section{GEOLOGICAL BACKGROUND}

The Dizi Series occurs in the core of the Svaneti anticlinorium, in the Enguri and Tskhenistskali river basins (Fig. 1). It is traceable for ca. $70 \mathrm{~km}$ in the Caucasian direction (north-west - south-east), with the maximum width of the outcropped area reaching $8 \mathrm{~km}$, and thickness $2000-2200 \mathrm{~m}$. It is mainly composed of weakly metamorphosed terrigenous sediments represented by strongly dislocated, crenulated and finely folded sandstones, gravel stones, argillites, carbonaceous-clayey shales, silicites, tuff sandstones, volcanic rocks and limestones (Fig. 2). Later, under the contact impact of the Middle Jurassic intrusions, they were transformed into graphitized phyllites, phyllitic schists, marbles and various hornfelses. In the Dizi Series, index fossils of corals and foraminifera of the Devonian, Carboniferous, Permian and Triassic ages were found (Slavin 1960; Somin, Belov 1967a; Somin 1971). Similar results were reported by Kutelia (1983), who was investigating conodonts. All these data prove that the Middle Paleozoic - Triassic stratigraphic section of the series is continuous (Somin 2011).

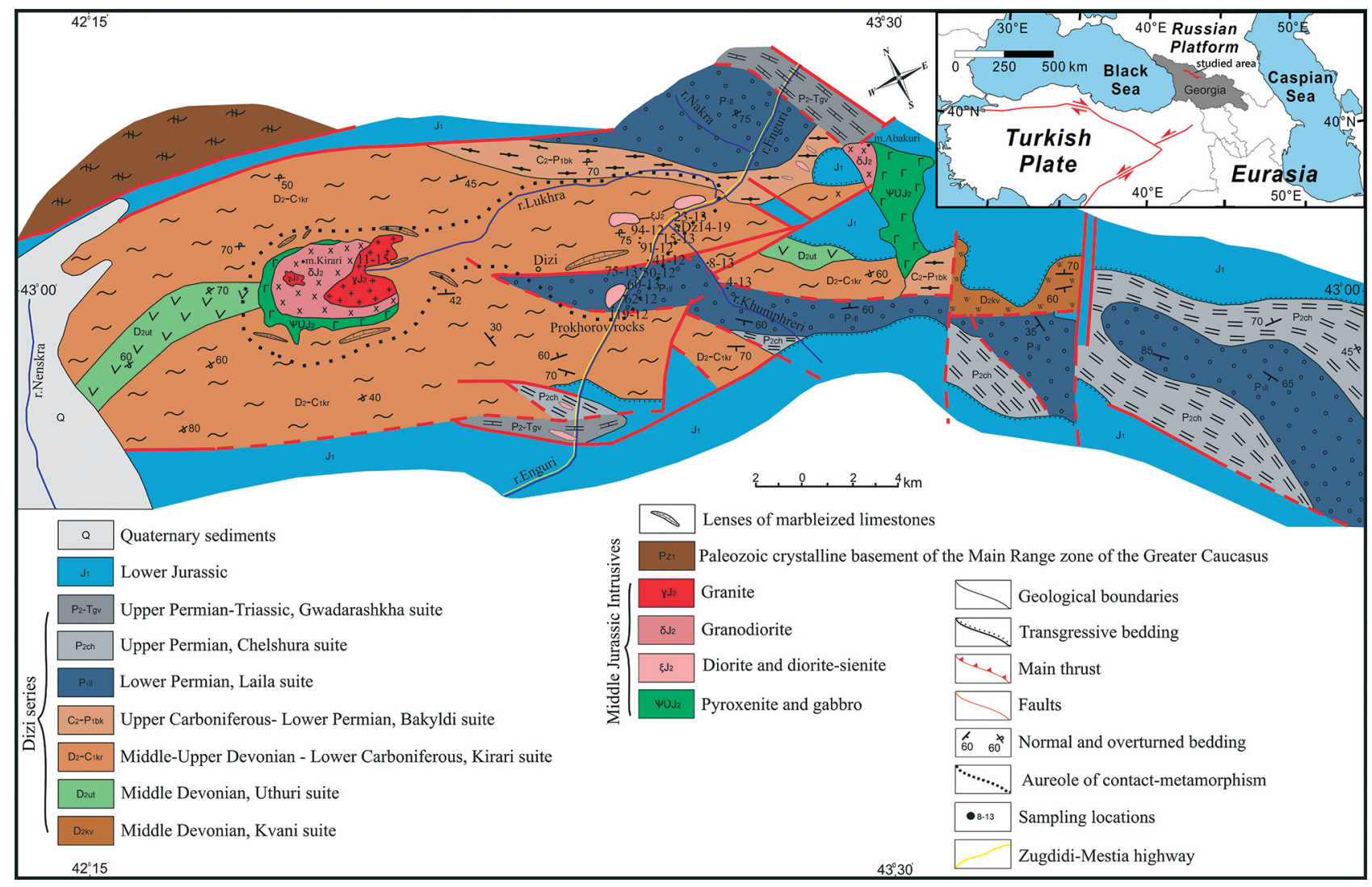

Fig. 1 Geological scheme of the northwestern part of the Svaneti anticlinorium (Somin, Belov 1967a, with additions by the authors) 


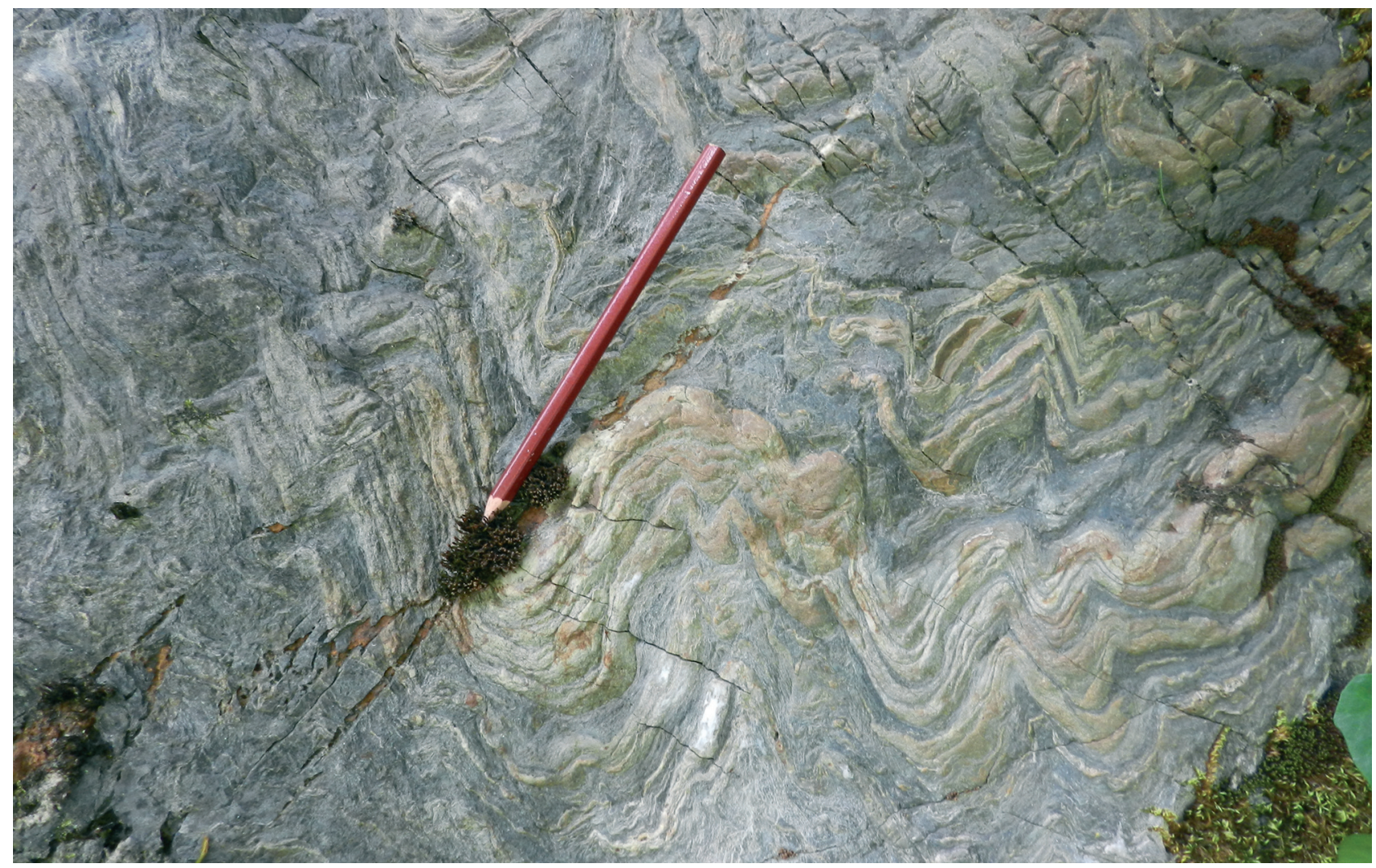

Fig. 2 Banded and folded sandy-clayey rocks. The gorge of the Khumpreri River, the left tributary of the river Enguri

The Late Triassic (Early Cimmerian) deformations are manifested in the sediments of the Dizi Series, which are deformed into highly compressed folds, complicated by fine folding and ruptures. Cleavage and faults are steeply dipping southwards. According to different authors (Kazmin, Sborshchikov 1989; Gamkrelidze, Shengelia 2005), the Dizi Series is generally characterized by an imbricate structure.

Considering the character of deformation, rocks of the Dizi Series sharply differ from the Jurassic deposits. They retain relict northern vergence, which indicates their movement to the north during the Early Cimmerian (Indosinian) orogeny, causing almost direct contact with highly metamorphosed rocks of the Main Range zone of the Greater Caucasus (Somin, Belov 1967a; Somin 1971; Gamkrelidze 1989; Belov 1986; Kazmin, Sborshchikov 1989). In the process of the overthrusting during the same orogeny, the dissipative heating of the Dizi Series rocks, folding, dynamometamorphism (Gamkrelidze, Shengelia 2005) and regional metamorphism (Somin 1971; Kazmin, Sborshchikov 1989; Shengelia et al. 2015) took place. Notably, Variscan tectonic events and granite formation did not take place in the Svaneti domain, in contrast to the crystalline basement of the Greater Caucasus, (Somin 1971; Kazmin, Sborshchikov 1989). During the Chegemian orogeny (Bathonian time), the eastern part of the relict Paleotethys basin was closed. These movements are clearly manifested in the north- ern part of the Mediterranean belt (the Greater Caucasus, the Mountainous Crimea, the Transcaucasian massif and the northern part of the Lesser Caucasus).

The Middle Jurassic epoch was marked by a powerful volcanism and magmatism. The Dizi Series hosts numerous bodies of pyroxenites, anorthosites, gabbro-diorites, diorites, quartz diorites, syenite-diorites, diorite-porphyrites and granitoids (Fig. 1). Kirari and Abakuri intrusions and intrusion in the environs of the Dizi village (3-5.5 $\left.\mathrm{km}^{2}\right)$, as well as numerous smaller intrusive bodies with thicknesses varying from one to $100 \mathrm{~m}$, were identified. These intrusions are embedded in the intensely deformed rocks of the Dizi Series, while the intrusions themselves are only slightly deformed, mainly in the form of rupture dislocations. Morphology of intrusive bodies, their spatial distribution, petrological and mineralogical similarities indicate that all of them are fragments of a single magma chamber (Goishvili 1960, 1961). The Kirari-Abakuri intrusive complex is mantle-derived and belongs to the calc-alkaline series (Dudauri, Togonidze 2016). It was contaminated with the crustal material. Most of the Bathonian intrusions are tholeiitic and belong to the group of continental and island arc magmatic rocks, and to the group of syncollisional magmatic rocks (Javakhishvili, Beridze 2020). The post-Liassic age of these intrusions was established geologically as rocks of the Abakuri intrusion cut both the Dizi Series and the Liassic sediments. The K-Ar age of 
these intrusions is $176-165 \mathrm{Ma}$ and corresponds to the Bathonian orogeny (Dudauri, Togonidze 2016).

Quartz-andalusite, quartz-biotite, biotite-chloritoid, quartz-potassium feldspar hornfelses and quartzites predominantly of crenulated structure (Supplement 1) develop at intrusive contacts with the Dizi Series rocks. Moving away from the contact, these rocks gradually turn into quartz-plagioclase-actinolite and sericite-chlorite schists (Goishvili 1960). Skarns, reaching the maximum thickness of 150-170 meters, develop in the places where intrusions contact with marbles.

It must be noted that the thickness of the intrusive bodies does not always correlate with that of their contact aureoles. The nature of the thermal effect is defined not only by the volume of the intruded magma, but also by its composition, amount of fluids, and the composition of host rocks. Fluid-rich granitoid (granodiorite, granite, and diorite-syenite) intrusions usually result in especially active transformation of host rocks, whereas relatively dry gabbro and gabbrodiorite intrusions are less efficient. Carbonates and sandy-clayey host rocks are the most vulnerable to these processes, while quartz sandstones and silicites are more inert (Goishvili 1960; Shengelia et al. 2015; Korikovsky et al. 2018; Javakhishvili et al. 2020).

\section{SAMPLE DESCRIPTION}

The material for this study was collected from the outcrops of the Dizi Series, covering contact aureoles around all Middle Jurassic intrusions, as well as regionally metamorphosed rocks (Fig. 1). Intrusive bodies and aureoles of contact metamorphism were mapped using GPS technology. Over 400 thin sections were studied under an optical microscope.

Rocks of regional metamorphism are represented by metamorphosed sandstones, gravel stones, argillites, carbonaceous shales, silicites, tuff sandstones, volcanic rocks, limestones, graphitized phyllites and phyllitic shales. Three samples of metasandstone, carbonate phyllite and phyllite alternating with graywacke were selected for electron microprobe analyses (Table 1, Supplement 2). Sample 4-13 is a carbonaceous chlo-

Table 1 Progressive transformations of minerals in metamorphic rocks of the Dizi Series

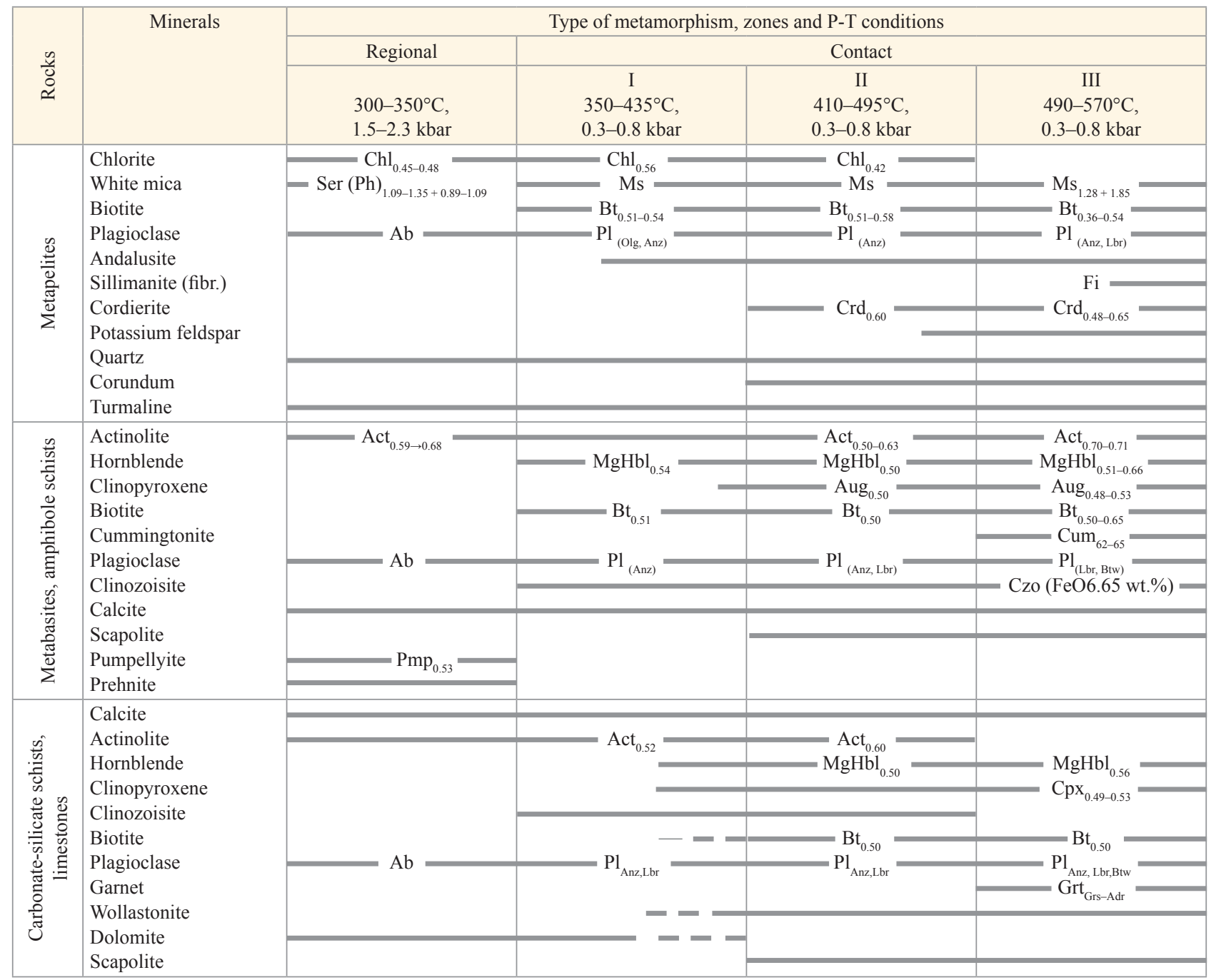


rite-sericite-albite-quartz metaaleurolite having blastoaleurolitic texture with microlepidoblastic cement; the structure is schistose-crenulated. Sample $8-13$ is a carbonaceous chlorite-sericite-albite-quartz phyllite with a microlepidoblastic texture and slaty structure. Sample 41-12 is an alternation of graphite-sericite-quartz phyllite with actinolite-prehnite-albite-quartz metagraywacke; the structure is streaky-micro-crenulated. In these rocks, pumpellyite was recorded once.

Ten samples representing contact-metamorphosed rocks (hornfelsed pelites, sandstones, basic rocks, amphibole and carbonate-silicate schists and marbles) were selected for electron microprobe analyses (see Table 1, Supplement 2). One of the rocks is a primary-sedimentary thin-banded rock with alternating fine-grained bands of felsic composition (bimica-plagioclase-quartz schist, sample 15-13) with $\mathrm{CaO}-$ rich melanocratic bands (hornblende-biotite-calcite-plagioclase-quartz graywacke, sample 15-13-1). Sample 23-13 represents graphite-bearing albite-bimica-andalusite porphyroblastic schist with a fabric characteristic of hornfels; sample 45-12 is calcite-actinolite-clinozoisite schist. All of the above rocks are hornfelsed, but they retain structural and textural features of the primary sedimentary rocks.

Further, according to the increasing recrystallization, samples 50-12, 50-12-1, 75-13 and 75-13-1 were selected. Sample 50-12 is porphyroblastic hornfels, composed of a small amount of chlorite and large andalusite crystals (sometimes forming radiating fibrous aggregates) and cordierite, filled with fine inclusions of biotite flakes and Na-poor muscovite. Sample 50-12-1 is hornfels with coexisting biotite, clinopyroxene, magnesian hornblende, actinolite, scapolite, and quartz. Sample 75-13 is thin-banded corundumbearing bimica-chlorite metapelite interlayered with sample 75-13-1. The latter is a rock with a mineral assemblage of biotite-clinopyroxene-hornblende-actinolite-clinozoisite-plagioclase-quartz with a mosaic texture and a heterogeneous structure.

The highest-temperature rocks are recorded at sites of direct contact with large intrusions (ca. $10 \mathrm{~m}$ ), as well as in xenoliths. Here, samples 119-12, 94-12, 91-12, 62-12, 11-15-2 and 11-15-3 were selected for analysis. The first three samples are plagioclasequartz-bimica-andalusite-cordierite hornfels, and sample 62-12 is garnet-scapolite-clinozoisite-pyroxene-biotite-plagioclase hornfels with idiomorphic porphyroblasts of hornblende. Samples 11-15-2 and 11-15-3 are xenoliths of basic hornfelses from the Kirari syenite-diorite intrusion; they are represented by amphibolites with the predominance of cummingtonite.

Sample Dz-14 represents an up to 10-metersthick diorite-porphyrite dyke, which crops out in the Khumpreri River gorge, the left tributary of the river
Enguri $\left(43^{\circ} 02^{\prime} 08.5^{\prime \prime} \mathrm{N} 42^{\circ} 21^{\prime} 22.9^{\prime \prime} \mathrm{E}\right)$. The rock contains phenocrysts of biotite, hornblende, and zoned plagioclase. The groundmass composed of the same minerals prevails over the phenocryst. Accessories are zircon, apatite and opaque minerals (Supplement 3). Zircon is a rare mineral in the magmatic rocks hosted by the Dizi Series, and it was found by the authors only in this sample.

\section{ANALYTICAL METHODS}

The composition of minerals (biotite, cordierite, muscovite, plagioclase, actinolite, actinolitic hornblende, hornblende, cummingtonite, clinopyroxene, chlorite, clinozoisite, K-feldspar, prehnite, pumpellyite and garnet) from all metamorphic rocks of the Dizi Series was analyzed in the Laboratory of Mineral Analysis of IGEM RAS using JEOL JXA-8200 electron Microprobe equipped with five wavelength dispersive X-ray spectrometers (WDS) and one energy-dispersive X-ray spectrometer (EDS). Analyses were performed at an accelerating voltage of $20 \mathrm{kV}$, beam current of $20 \mathrm{nA}$ and beam size of $1-2 \mu \mathrm{m}$ with counting times of $10 \mathrm{~s}$. All elements were measured along the Ka1 analytical line. Results of the analyses are presented in Supplement 2.

Various geothermometers were applied to determine P-T conditions of the regional and contact metamorphism of the Dizi Series. The use of certain geothermometers is conditioned by the presence of equilibrium mineral parageneses in regional and contact metamorphic rocks of the Dizi Series, as well as by microprobe analysis data. To define temperature conditions of regional metamorphism, low-temperature chlorite thermometers were used according to various authors (Cathelineau, Nieva 1985; Kranidiotis, McLean 1987; Cathelineau 1988; Jowett 1991; Hiller, Velde 1991; Zang, Fyfe 1995). Higher temperatures of contact metamorphism were established using the following geothermometers: Hbl-Pl (Holland, Blundy 1994; Jaques et al. 1982; Spear 1981), Bt-Cpx and Hbl-Cpx (Perchuk, Ryabchikov 1976), Crd-Bt (Lavrentyeva, Perchuk 1981) and Ti in Ms (Chun-Ming Wu, Hong-Xu Chen 2015). The degree of metamorphism in rocks is assessed based on a number of factors including characteristic mineral associations, general considerations, the informative scheme of metamorphic facies of regional metamorphism adopted in this work (Korikovsky 1991), the gradient model elaborated for the pre-Alpine metamorphic complexes of the Greater Caucasus (Korikovsky et al. 1991), and comparison of the region with other well-studied areas (Reverdatto 1970; Korikovsky et al. 1984, 1986).

The age of the Dizi Series intrusive rocks was determined using U-Pb LA-ICP-MS zircon dating. 
Zircon crystals were extracted from crushed samples using standard separation techniques (shaking table, magnetic separation and heavy liquids) at the M.P. Semenenko Institute of Geochemistry, Mineralogy and Ore Formation, Kyiv, the Ukraine. The crystals were hand-picked under an optical microscope, mounted in epoxy resin and polished to expose their internal parts. Zircon was ablated using a COMPex 102-193 nm excimer UV laser coupled with an Agilent 8900 QQQ mass spectrometer. After $30 \mathrm{~s}$ of background analysis, the samples were spot ablated for $30 \mathrm{~s}$ at $5 \mathrm{~Hz}$ using a $38 \mu \mathrm{m}$ beam and laser energy at the sample surface of $2 \mathrm{~J} \mathrm{~cm}^{-2}$. The sample cell was flushed with ultrahigh purity $\mathrm{He}$ and $\mathrm{N}_{2}$ and high purity Ar was employed as a plasma carrier gas. Zircon standard GJ1 (601.2 $\pm 0.4 \mathrm{Ma}$; Jackson et al. 2004) was utilized as the primary reference material, and analysed in blocks with secondary standards Plešovice (337.13 $\pm 0.37 \mathrm{Ma}$; Sláma et al. 2008) and OGC (3465 $\pm 0.6 \mathrm{Ma}$; Stern et al. 2009). Standard blocks were inserted between every 20 unknowns. The secondary standards yielded the weighted mean ${ }^{207} \mathrm{~Pb} /{ }^{206} \mathrm{~Pb}$ ages and ${ }^{238} \mathrm{U} /{ }^{206} \mathrm{~Pb}$ ages within uncertainty of the recommended values.

Data were collected in the course of two analytical sessions. In the first session, only U-Pb isotope data were collected. The ${ }^{206} \mathrm{~Pb} /{ }^{204} \mathrm{~Pb}$ ratio values estimated during this session were rather low, but generally concordant. During the second session (in Table 2, the results are marked with the letter "a"), the U-Pb data were collected simultaneously with Hf isotopes and trace elements (split-stream technology). The $\mathrm{Lu}-$ $\mathrm{Hf}$ isotope composition was measured at the John de Laeter Centre, Curtin University, Australia, using a $\mathrm{Nu}$ Plasma II mass-spectrometer. All isotopes $\left({ }^{180} \mathrm{Hf}\right.$, ${ }^{179} \mathrm{Hf},{ }^{178} \mathrm{Hf},{ }^{177} \mathrm{Hf},{ }^{176} \mathrm{Hf},{ }^{175} \mathrm{Lu},{ }^{174} \mathrm{Hf},{ }^{173} \mathrm{Yb},{ }^{172} \mathrm{Yb}$ and ${ }^{171} \mathrm{Yb}$ ) were counted using the Faraday collector array. Contributions of ${ }^{176} \mathrm{Yb}$ and ${ }^{176} \mathrm{Lu}$ were removed from the 176 mass signal using ${ }^{176} \mathrm{Yb} /{ }^{173} \mathrm{Yb}=0.7962$ and ${ }^{176} \mathrm{Lu} /{ }^{175} \mathrm{Lu}=0.02655$ with an exponential-law mass bias correction assuming that ${ }^{172} \mathrm{Yb} /{ }^{173} \mathrm{Yb}=1.35274$ (Chu et al. 2002). In each session, zircon crystals from the Mud Tank carbonatite were analyzed together with the samples so as to monitor the accuracy of results. Zircons 91500; Plešovice; GJ-1 and R33 were also run as secondary reference standards. All reference material yielded ${ }^{176} \mathrm{Hf} /{ }^{177} \mathrm{Hf}$ ratios within uncertainty of their respective reported values. Initial ${ }^{176} \mathrm{Hf} /{ }^{177} \mathrm{Hf}$ and $\varepsilon \mathrm{Hf}$ values for unknown zircons were calculated employing the measured ${ }^{207} \mathrm{~Pb} /{ }^{206} \mathrm{~Pb}$ spot date; a $\lambda^{176} \mathrm{Lu}$ decay constant of $1.867 \times 10^{-11}$ (Söderlund et al. 2004); and a present-day Chondritic Uniform Reservoir (CHUR) ${ }^{176} \mathrm{Hf} /{ }^{177} \mathrm{Hf}=0.282785$ and ${ }^{176} \mathrm{Lu} /{ }^{177} \mathrm{Hf}=0.0336$ (Bouvier et al. 2008).

Trace element data were collected simultaneously with Hf isotope data. Zircon standard GJ-1 was uti- lized as the primary reference material for determining concentration and correcting instrument drift, using ${ }^{91} \mathrm{Zr}$ as an internal reference isotope and assuming that $\mathrm{Zr}$ percentage in unknowns is $43.14 \%$. NIST SRM 612 was run as a secondary standard, yielding recommended values within 3\% for all elements.

\section{RESULTS}

The regionally metamorphosed rocks of the Dizi Series are represented by metaterrigenous phyllitelike and volcanogenic rocks and marbles. Their main minerals are sericite (phengite), albite, chlorite, actinolite, calcite, tourmaline, dolomite, pumpellyite, and prehnite (Supplement 2).

According to the results of electron-microprobe analysis, the key mineral associations of the regional metamorphism in the Dizi Series rocks are: $\mathrm{Chl}_{0.48}+$ $\operatorname{Ser}\left(\mathrm{Ph}_{2.01+1.99}\right)+\mathrm{Ab}+\mathrm{Qz} \pm$ Tur $^{1}$ (sample 4-13, metaaleurolite) and $\mathrm{Chl}_{0.45}+\mathrm{Ser}\left(\mathrm{Ph}_{1.3+1.0}\right)+\mathrm{Cal}+\mathrm{Ab}+\mathrm{Qz}$ (sample 8-13, carbonaceous phyllite). Fine intercalation of graphite-sericite-quartz phyllites with Act $_{0.59}$ $+\mathrm{Phr}+\mathrm{Ab} \pm \mathrm{Qz}$ greywacke was also observed. As mentioned above, pumpellyite was noted in a single case $\mathrm{Act}_{0.59}+\mathrm{Pmp}_{0.53}+\mathrm{Phr}+\mathrm{Ab}+\mathrm{Qz}$ (sample 41-12). Especially noteworthy is the presence of fresh detrital grains of augite $\left(\operatorname{Aug}_{0.55}\right)$ and $\mathrm{Mg}$-hornblende $(\mathrm{Mg}$ $\mathrm{Hbl})_{0.60: 0.62}$ in sample 41-12. A similar case was reported from the Taveyanne Formation in Switzerland (Coombs et al. 1976). The mineral composition of regional metamorphic rocks is presented in Supplement 2. The temperature of regional metamorphism determined using low-temperature chlorite thermometer was $296-366{ }^{\circ} \mathrm{C}$ (Cathelineau, Nieva 1985; Kranidiotis, McLean 1987; Cathelineau 1988; Jowett 1991; Hiller, Velde 1991; Zang, Fyfe 1995).

Previously deformed and regionally metamorphosed rocks of the Dizi Series underwent contact metamorphism (Supplement 4). The width of the contact aureole reaches ca. 350 meters. The most extensive outcrops of the contact-metamorphosed rocks stretch along the Zugdidi-Mestia highway for up to $5000 \mathrm{~m}$ between the Prokhorov rocks and the Lukhra River (the right tributary of the river Enguri). It should be mentioned that this stretch hosts numerous unevenly distributed intrusive bodies of different size. The effect of their contact on host rocks is also uneven. In some places, regionally metamorphosed rocks have remained completely unaffected by the contact alteration. Occasionally, high-temperature

\footnotetext{
1 Mineral symbols are given as per Whitney, Evans (2010). Classification of amphiboles is given according to Hawthorne et al. (2012). Index numbers of dark-colored minerals indicate their magnesium numbers $\left(\mathrm{Mg} /\left(\mathrm{Mg}+\mathrm{Fe}^{2+}\right)\right)$; arrows indicate variations in magnesium numbers from the center to the periphery of Ca-amphibole crystals; index numbers of $\mathrm{Pl}$ are the content of the anorthite molecule, and Ms indices indicate the $\mathrm{FeO}+\mathrm{MgO}$ value (wt. \%).
} 


\begin{tabular}{|c|c|c|c|c|c|c|c|c|c|c|c|c|c|c|c|c|c|c|c|}
\hline \multirow{4}{*}{ 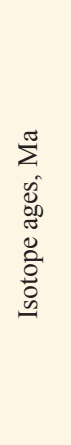 } & $\stackrel{0}{\sim}$ & $\approx$ & 5 & $\cong$ & $\tilde{\lambda}$ & \pm & in & in & రু & $\approx$ & 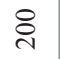 & $\stackrel{\circ}{\ni}$ & $\vec{n}$ & $\stackrel{8}{ }$ & $\triangleright$ & $\stackrel{\circ}{m}$ & in & $\stackrel{\infty}{\stackrel{\infty}{+}}$ & $\underset{ల}{8}$ \\
\hline & 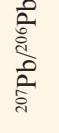 & $\overline{\mathcal{Y}}$ & જे & તิ તิ & $\stackrel{\infty}{\infty}$ & 吕 & 孛 & gु & $\underset{\mathscr{\infty}}{\stackrel{⿰}{\alpha}}$ & î̀ & 옹 & 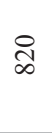 & స્ત & $\stackrel{\infty}{\stackrel{\infty}{\alpha}}$ & $\tilde{\mathscr{O}}$ & 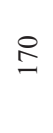 & 品 & ㄹ & $\stackrel{\infty}{\stackrel{\infty}{\sigma}}$ \\
\hline & $\stackrel{0}{\sim}$ & $\ddot{n}$ & $\stackrel{m}{?}$ & $\bar{m}$ & $\sim$ & $\stackrel{0}{i}$ & $\stackrel{0}{0}$ & $=$ & $\stackrel{2}{r}$ & $=$ & $\stackrel{m}{\infty}$ & $\stackrel{\infty}{=}$ & $\mathbb{尺}$ & $m$ & $\vec{i}$ & \pm & $=$ & $\stackrel{\infty}{\sim}$ & I \\
\hline & 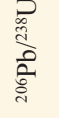 & $\begin{array}{l}0 \\
\dot{n} \\
\dot{q}\end{array}$ & ㄹ. & $\begin{array}{l}\tilde{\mathbb{N}} \\
\tilde{N}\end{array}$ & $\stackrel{\bullet}{\circ}$ & \begin{tabular}{l} 
for \\
\multirow{d}{*}{}
\end{tabular} & $\begin{array}{l}\infty \\
\dot{o} \\
\dot{+}\end{array}$ & శ্ণ & $\begin{array}{l}n \\
\ddot{y} \\
y \\
y\end{array}$ & $\stackrel{\leftrightarrow}{\sigma}$ & $\overrightarrow{\vec{g}}$ & $\stackrel{m}{m}$ & $\underset{\sim}{\Xi}$ & $\begin{array}{l}0 \\
\infty \\
\infty \\
n\end{array}$ & ठें & 角 & ì & ర్ల & 品 \\
\hline \multirow{11}{*}{ 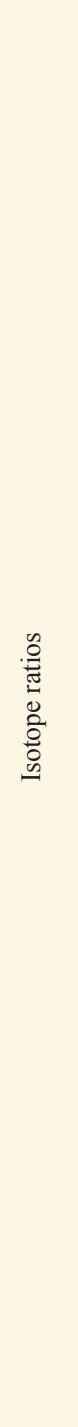 } & 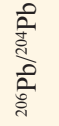 & $\ddot{\sim}$ & $\vec{\sim}$ & in & $\stackrel{\infty}{\beth}$ & 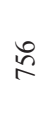 & $\approx$ & $\approx$ & $\grave{I}$ & F & $\begin{array}{l}\stackrel{\Delta}{ } \\
i\end{array}$ & $\begin{array}{l}\stackrel{8}{0} \\
\vdots \\
+\end{array}$ & $\begin{array}{l}\text { ڤ } \\
\text { }\end{array}$ & 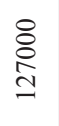 & $\begin{array}{l}\stackrel{0}{\infty} \\
\stackrel{\infty}{\sim}\end{array}$ & $\begin{array}{l}\text { ¿े } \\
\text { הి }\end{array}$ & $\underset{0}{\circ}$ & 各 & $\begin{array}{l}\stackrel{8}{\circ} \\
\stackrel{\infty}{+}\end{array}$ \\
\hline & $a$ & $\vec{J}$ & గి. & $\stackrel{0}{\stackrel{0}{0}}$ & $\stackrel{f}{0}$ & n̊. & $\stackrel{\infty}{+}$ & $\vec{b}$ & $\stackrel{m}{\stackrel{0}{0}}$ & $\stackrel{8}{\circ}$ & J. & กิ & $\begin{array}{l}\stackrel{+}{1} \\
\stackrel{i}{i}\end{array}$ & $=$ & $\stackrel{8}{0}$ & $\stackrel{े}{0}$ & 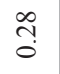 & $\stackrel{\circ}{\circ}$ & $\stackrel{n}{\text { ?f. }}$ \\
\hline & $\stackrel{0}{\sim}$ & $\stackrel{m}{\stackrel{m}{o}}$ & $\begin{array}{l}\overline{\mathrm{\sigma}} \\
\stackrel{0}{\circ}\end{array}$ & $\begin{array}{l}\overrightarrow{\tilde{o}} \\
\stackrel{0}{\circ}\end{array}$ & $\begin{array}{l}\vec{\Xi} \\
\dot{\circ}\end{array}$ & $\overrightarrow{\bar{o}}$ & $\overrightarrow{\tilde{o}}$ & $\begin{array}{l}\infty \\
\stackrel{\infty}{0} \\
0\end{array}$ & $\begin{array}{l}\text { ठิ } \\
\text { ळ. }\end{array}$ & 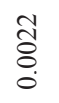 & $\begin{array}{l}0 \\
\stackrel{2}{0} \\
0 \\
0\end{array}$ & $\begin{array}{l}\stackrel{2}{0} \\
\stackrel{0}{0}\end{array}$ & 宅 & \begin{tabular}{l}
0 \\
\multirow{0}{0}{} \\
0 \\
0
\end{tabular} & $\begin{array}{l}\text { ठे } \\
\stackrel{0}{0}\end{array}$ & $\begin{array}{l}\text { oे } \\
\text { oे }\end{array}$ & $\begin{array}{l}0 \\
\stackrel{\infty}{0} \\
0\end{array}$ & $\stackrel{8}{\circ}$ & $\stackrel{\text { P) }}{\stackrel{0}{0}}$ \\
\hline & 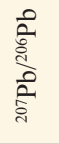 & 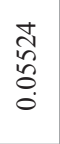 & 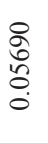 & $\frac{\stackrel{\circ}{\vec{I}}}{\stackrel{\mathrm{I}}{0}}$ & $\begin{array}{l}\vec{\infty} \\
\dot{0} \\
\dot{0} \\
0\end{array}$ & 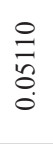 & $\begin{array}{l}0 \\
0 \\
o \\
o \\
0 \\
0\end{array}$ & $\begin{array}{l}\stackrel{2}{n} \\
\tilde{n} \\
0 \\
0\end{array}$ & $\begin{array}{l}8 \\
0 \\
0 \\
0 \\
0\end{array}$ & $\begin{array}{l}0 \\
\stackrel{n}{n} \\
\hat{o} \\
0 \\
0\end{array}$ & $\begin{array}{l}\text { ơ } \\
0 \\
0 \\
0 \\
0\end{array}$ & $\begin{array}{l}\stackrel{\circ}{0} \\
\infty \\
\stackrel{0}{0}\end{array}$ & $\begin{array}{l}\stackrel{8}{\infty} \\
\stackrel{0}{0} \\
0 \\
0\end{array}$ & 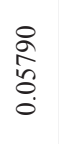 & $\begin{array}{l}0 \\
\stackrel{0}{0} \\
\stackrel{్}{0} \\
\stackrel{0}{0}\end{array}$ & 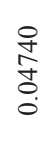 & $\underset{:}{\stackrel{8}{\circ}}$ & $\begin{array}{l}8 \\
0 \\
\tilde{o} \\
0 \\
0\end{array}$ & $\begin{array}{l}8 \\
\& \\
\stackrel{0}{0} \\
\stackrel{0}{0}\end{array}$ \\
\hline & $\stackrel{0}{\sim}$ & 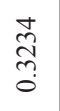 & 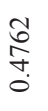 & $\begin{array}{l}0 \\
0 \\
0 \\
0\end{array}$ & $\begin{array}{l}\hat{\sigma} \\
\text { مे } \\
\text { o. }\end{array}$ & $\begin{array}{l}\bar{\partial} \\
\text { oे } \\
\text { o }\end{array}$ & $\begin{array}{l}\text { đ̃ } \\
\text { ఫे } \\
0\end{array}$ & $\begin{array}{l}\text { Ẽ } \\
\hat{0} \\
0\end{array}$ & $\begin{array}{l}0 \\
\infty \\
0 \\
0 \\
0\end{array}$ & 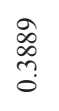 & $\begin{array}{l}\overrightarrow{0} \\
\stackrel{\infty}{0} \\
\stackrel{0}{0}\end{array}$ & 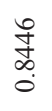 & $\begin{array}{l}\bar{\sigma} \\
\text { Oे }\end{array}$ & $\begin{array}{l}0 \\
\stackrel{0}{0} \\
0 \\
0\end{array}$ & $\frac{\hat{a}}{3}$ & $\begin{array}{l}\bar{N} \\
\hat{n} \\
\vdots\end{array}$ & $\frac{\widetilde{\partial}}{\stackrel{\partial}{0}}$ & $\begin{array}{l}\text { dे } \\
\text { कo } \\
0\end{array}$ & 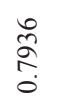 \\
\hline & 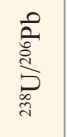 & 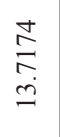 & $\begin{array}{l}\infty \\
\stackrel{\infty}{\infty} \\
\stackrel{0}{\sigma} \\
\underline{6}\end{array}$ & $\begin{array}{l}\text { ते } \\
\text { ले } \\
\text { i }\end{array}$ & 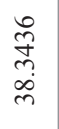 & 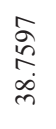 & $\begin{array}{l}\stackrel{0}{0} \\
\stackrel{0}{2} \\
\stackrel{2}{2}\end{array}$ & $\begin{array}{l}\tilde{\hat{D}} \\
\text { I } \\
\text { - }\end{array}$ & 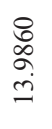 & $\begin{array}{l}\stackrel{0}{n} \\
\stackrel{n}{n} \\
=\end{array}$ & $\begin{array}{l}\underset{J}{ \pm} \\
\underset{ \pm}{ \pm}\end{array}$ & $\begin{array}{l}\infty \\
\stackrel{\infty}{8} \\
\stackrel{0}{6} \\
\stackrel{0}{0}\end{array}$ & $\begin{array}{l}\hat{a} \\
\hat{n} \\
i\end{array}$ & 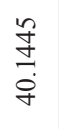 & $\begin{array}{l}\text { Uै } \\
0 \\
0 \\
0 \\
0\end{array}$ & 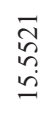 & $\begin{array}{l}\stackrel{D}{\infty} \\
\stackrel{\infty}{i} \\
\stackrel{n}{n}\end{array}$ & 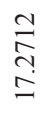 & 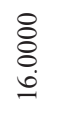 \\
\hline & a & $\stackrel{n}{0}$ & i. & $\stackrel{0}{:}$ & 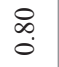 & $\stackrel{0}{0}$ & f. & $\stackrel{\vec{\infty}}{\overrightarrow{0}}$ & ల్ర & $\stackrel{\because}{0}$ & $\stackrel{n}{0}$ & $\bar{m}$ & $\stackrel{\substack{\infty \\
\infty}}{0}$ & $\stackrel{7}{0}$ & $\stackrel{\infty}{\stackrel{0}{0}}$ & $\stackrel{n}{0}$ & $\stackrel{\infty}{\circ}$ & ָָ & $\stackrel{\overline{0}}{\circ}$ \\
\hline & $\stackrel{b}{\sim}$ & $\stackrel{\bar{\sigma}}{\circ}$ & 훙 & $\stackrel{\circ}{\stackrel{0}{0}}$ & $\begin{array}{l}\text { ¿े } \\
0 \\
0\end{array}$ & $\begin{array}{l}\text { ò } \\
0\end{array}$ & 홍 & $\begin{array}{l}\bar{m} \\
\stackrel{0}{0}\end{array}$ & $\stackrel{\vec{\partial}}{\circ}$ & 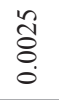 & $\begin{array}{l}\vec{\Delta} \\
\overrightarrow{8} \\
\dot{0}\end{array}$ & $\begin{array}{l}0 \\
\stackrel{0}{0} \\
0 \\
0\end{array}$ & $\stackrel{\circ}{\stackrel{n}{0}}$ & $\begin{array}{l}\text { oे } \\
\stackrel{0}{0}\end{array}$ & $\begin{array}{l}\text { ôे } \\
\text {. }\end{array}$ & $\begin{array}{l}\text { ธे } \\
\text {. }\end{array}$ & ठे & $\begin{array}{l}\text { \%̊̊ } \\
\stackrel{0}{0}\end{array}$ & के \\
\hline & 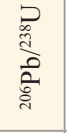 & $\begin{array}{l}\text { సे } \\
\text { ¿े. }\end{array}$ & $\begin{array}{l}\text { \&े } \\
\text { ò. } \\
0\end{array}$ & 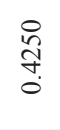 & 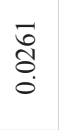 & $\begin{array}{l}\infty \\
\stackrel{2}{0} \\
0 \\
0\end{array}$ & $\begin{array}{l}\overrightarrow{\tilde{b}} \\
\stackrel{0}{0}\end{array}$ & $\begin{array}{l}\overrightarrow{0} \\
\text { Oे. } \\
0\end{array}$ & $\frac{n}{a}$ & $\begin{array}{l}\text { ôे } \\
\text { Oे. }\end{array}$ & $\begin{array}{l}n \\
0 \\
0 \\
0 \\
0\end{array}$ & $\begin{array}{l}\text { \&̊ } \\
\text { oे } \\
0\end{array}$ & $\begin{array}{l}0 \\
\text { o } \\
\text { co } \\
0\end{array}$ & $\begin{array}{l}\text { वे. } \\
\text { d. } \\
\text { Oे }\end{array}$ & 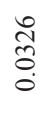 & 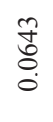 & 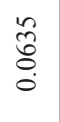 & $\begin{array}{l}\hat{y} \\
\hat{s} \\
0\end{array}$ & $\begin{array}{l}\text { ते } \\
\text { ठ̊. } \\
\text {. }\end{array}$ \\
\hline & $\stackrel{\bullet}{\sim}$ & 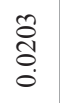 & $\begin{array}{l}\overline{\widetilde{\sigma}} \\
\stackrel{0}{\circ}\end{array}$ & 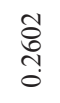 & $\begin{array}{l}\text { tơ } \\
\text { ¿ } \\
0\end{array}$ & & $\begin{array}{l}\stackrel{\partial}{\partial} \\
\dot{0}\end{array}$ & $\begin{array}{l}\text { त̂ } \\
\text { Oे }\end{array}$ & $\begin{array}{l}\text { तิ } \\
\text { ठ }\end{array}$ & $\begin{array}{l}\tilde{\mathscr{N}} \\
\text { } \\
\stackrel{0}{0}\end{array}$ & $\begin{array}{l}0 \\
\stackrel{0}{0} \\
\stackrel{0}{0}\end{array}$ & 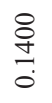 & $\begin{array}{l}\stackrel{8}{\infty} \\
\substack{0 \\
0}\end{array}$ & $\frac{\text { P }}{\stackrel{0}{0}}$ & $\stackrel{8}{\circ}$ & $\begin{array}{l}\text { : } \\
\stackrel{0}{0}\end{array}$ & $\begin{array}{l}8 \\
\stackrel{n}{0} \\
0\end{array}$ & $\begin{array}{l}\stackrel{0}{1} \\
0 \\
0\end{array}$ & $\frac{8}{\circ}$ \\
\hline & 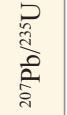 & $\begin{array}{l}0 \\
\hat{n} \\
\vdots \\
0\end{array}$ & \begin{tabular}{l} 
गें \\
\multirow{0}{0}{}
\end{tabular} & 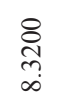 & $\begin{array}{l}\bar{\Xi} \\
\stackrel{0}{0}\end{array}$ & $\begin{array}{l}\stackrel{0}{0} \\
\stackrel{\infty}{0}\end{array}$ & $\frac{8}{n}$ & $\begin{array}{l}\infty \\
\tilde{n} \\
\tilde{n}\end{array}$ & $\begin{array}{l}\infty \\
0 \\
\hat{n} \\
0\end{array}$ & 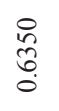 & \begin{tabular}{l}
0 \\
\multirow{d}{*}{} \\
0 \\
0
\end{tabular} & $\begin{array}{l}8 \\
\text { : } \\
0 \\
0\end{array}$ & 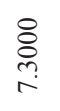 & $\frac{n}{2}$ & $\begin{array}{l}0 \\
\stackrel{0}{0} \\
\stackrel{0}{0}\end{array}$ & 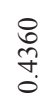 & $\begin{array}{l}\text { ڤे } \\
\text { م. }\end{array}$ & $\begin{array}{c}\stackrel{8}{\infty} \\
\stackrel{\infty}{0} \\
0\end{array}$ & $\begin{array}{l}\text { o. } \\
\text { n. } \\
0\end{array}$ \\
\hline$\stackrel{?}{?}$ & & $\underset{\sim}{\stackrel{\delta}{-}}$ & 足 & గ̂. & 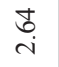 & : & : & $n$ & $\vec{n}$ & ?q & $\stackrel{\simeq}{\leftrightarrows}$ & : & 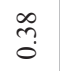 & $\stackrel{\substack{\infty \\
-}}{.}$ & $\underset{i}{\stackrel{N}{r}}$ & $\bar{a}$ & ñ & $\sqrt[n]{n}$ & రై \\
\hline \multirow{2}{*}{ 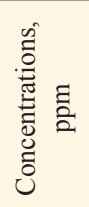 } & $F$ & 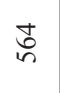 & $\tilde{\infty}$ & $\triangleright$ & $\stackrel{n}{\vec{n}}$ & 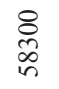 & $\cong$ & $\infty$ & $\tilde{\infty}$ & is & $\stackrel{0}{n}$ & $\alpha$ & ถิ & $\stackrel{8}{\Rightarrow}$ & $\begin{array}{l}\stackrel{8}{\infty} \\
\text { i } \\
\text { N }\end{array}$ & ते & q & $\stackrel{\infty}{+}$ & 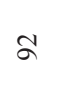 \\
\hline & D & $\begin{array}{l}n \\
i n\end{array}$ & 吉 & $\stackrel{n}{n}$ & 乎 & $\begin{array}{l}80 \\
\text { on } \\
\end{array}$ & $\cong$ & $\underline{n}$ & 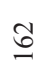 & $\alpha$ & \& & ले & $\stackrel{\infty}{\vec{f}}$ & $\stackrel{\circ}{\stackrel{\sim}{\sim}}$ & 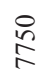 & ָั & 尺 & $\Xi$ & $\stackrel{\infty}{ \pm}$ \\
\hline & $\begin{array}{l}\stackrel{f}{+} \\
\text { N่ }\end{array}$ & 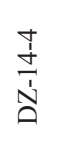 & $\frac{n}{\stackrel{n}{ \pm}}$ & $\begin{array}{l}\text { I } \\
\text { İ } \\
\text { N. }\end{array}$ & $\frac{\mathfrak{I}}{\hat{N}}$ & 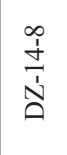 & $\begin{array}{l}\stackrel{a}{\dot{I}} \\
\stackrel{\hat{N}}{a}\end{array}$ & $\begin{array}{l}\frac{7}{d} \\
\frac{1}{1} \\
\text { an }\end{array}$ & $\begin{array}{l}\frac{d}{d} \\
\frac{\vec{N}}{0}\end{array}$ & 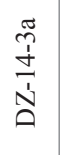 & $\begin{array}{l}\frac{\mathfrak{g}}{+} \\
\frac{1}{\hat{N}}\end{array}$ & 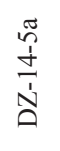 & 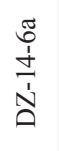 & $\begin{array}{l}\frac{\pi}{d} \\
\frac{\pi}{d} \\
\hat{N}\end{array}$ & 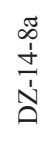 & 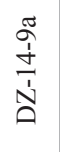 & 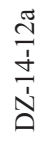 & $\begin{array}{l}\frac{\pi}{ \pm} \\
\dot{J} \\
\text { N่ }\end{array}$ \\
\hline
\end{tabular}


hornfelses occur in places where intrusive bodies do not crop out, indicating that igneous rocks are located close to the surface. The mutual overlap of the contact-metamorphic zones produced by different intrusive bodies significantly complicates metamorphic zoning, because in separate zones, it hinders thickness determination. Numerous rupture dislocations also play an important role in distorting the primary picture of metamorphic zoning.

The beginning of the contact alteration in regionally metamorphosed rocks is marked by the appearance of biotite, muscovite and oligoclase-andesine in metapelites, hornblende, biotite and clinozoisite in metabasites and amphibole schists, and wollastonite and clinozoisite in carbonate-silicate schists (Table 1). The following mineral associations can be distinguished (Supplement 2): $\mathrm{Bt}_{054}+\mathrm{Ms}_{135+0.89: 175+1.99}$ $+\mathrm{Pl}_{0.46 \text { (Anz) }}+\mathrm{Qz}$ (sample 15-13) and $\mathrm{MgHbl}_{0.54}+\mathrm{Bt}_{0.51}$ $+\mathrm{Cal}+\mathrm{Pl}_{0.82}+\mathrm{Qz}$ (sample 15-13-1); Bt $+\mathrm{Ms}+$ And $+\mathrm{Ab} \pm \mathrm{Qz} \pm \mathrm{Chl}_{0.56}\left(\right.$ sample 23-13); $\mathrm{Cal}+\mathrm{Act}_{0.52}+$ Czo (sample 45-12) and $\mathrm{Cal}+\mathrm{Cpx}+\mathrm{Wo}+\mathrm{Gr}+\mathrm{Qz}$ (sample 43-12).

Closer to intrusion bodies, as well as at sites of direct contact with thin intrusions, the degree of recrystallization increases. As a rule, relics of the primary minerals are no longer present, although their structural features are still preserved; cordierite and corundum appear in metapelites, whereas scapolite and clinopyroxene are widely distributed in metabasites and in amphibole and carbonate-silicate schists. The minerals characteristic of metapelites are andalusite, biotite, plagioclase, corundum, rarely cordierite, muscovite, and chlorite. Due to low-pressure conditions, garnet is not found, but an alternative association of chlorite + quartz + magnetite is widely distributed. Rocks rich in $\mathrm{CaO}$ are composed of hornblende, actinolite, clinopyroxene, minerals of the epidote group, plagioclase and biotite, whereas quartz, scapolite and calcite are rare minerals. The characteristic mineral association in metapelites (sample 50-12) is And +
$\mathrm{Bt}_{0.51-0.58}+\mathrm{Crd}_{0.60}+\mathrm{Chl}_{0.42}+\mathrm{Ms}_{1.09+1.09}+\mathrm{Pl}_{0.34-0.35}+$ Mag $+( \pm$ Crn or Qz). This rock is the porphyroblastic schist, composed of a small amount of chlorite and large crystals of andalusite (sometimes forming radiating fibrous aggregate) and cordierite, filled with fine inclusions of biotite flakes and Na-poor muscovite. The mineral association $\mathrm{Cpx}+\mathrm{MgHbl}_{0.50}+\mathrm{Bt}_{0.50}$ + Act + Scp was identified in Sample 50-12-1. Mineral association in sample 75-13 is $\mathrm{Bt}+\mathrm{Ms}+\mathrm{Crn}+$ $\mathrm{Chl} \pm \mathrm{Qz}$, but in sample 75-13-1, it is $\mathrm{Cpx}_{0.50}+\mathrm{Hbl}+$ $\mathrm{Act}_{0.50-0.63}+\mathrm{CzO}+\mathrm{Pl}_{0.41 ;: 0.56 ; 0.77}+\mathrm{Bt}_{0.50} \pm \mathrm{Qz}$. To determine temperature conditions of the contact metamorphism of the above-mentioned rocks, an Hbl-Pl geothermometer was applied and the temperature range of $436-503^{\circ} \mathrm{C}$ was estimated (Holland, Blundy 1994; Jaques et al. 1982; Spear 1981).

Still closer to intrusive bodies, approximately at a distance of $10 \mathrm{~m}$ from the contact site, as well as in xenoliths, the highest-temperature rocks are recorded. The main changes in metapelites include the disappearance of chlorite and formation of fibrolite (after biotite) (Fig. 3), whereas cummingtonite develops in metabasites. Garnet appears in carbonate-silicate schists, while actinolite and clinozoisite disappear.

The following mineral associations were identified in metapelites: sample 119-12: And $+\mathrm{Bt}_{0.46}+$ $\mathrm{Crd}_{0.64-0.65}+\mathrm{Ms}_{1.01+0.96,1.28+1.28} \pm \mathrm{Pl}+\mathrm{Qz}$ (Supplement $5-\mathrm{a}, \mathrm{b})$, sample 94-12: And $+\mathrm{Bt}_{0.36}(\rightarrow \mathrm{Fi})+\mathrm{Crd}_{048}$ $+\mathrm{Ms}_{0.90+0.38,1.02+0.44} \pm \mathrm{Pl}+\mathrm{Kfs}+\mathrm{Qz}$ (Supplement 5$\mathrm{c}, \mathrm{d})$ and sample 91-12: And $+\mathrm{Bt}_{0.36-0.40}+\mathrm{Crd}_{0.48-0.50}$ $+\mathrm{Ms} \pm \mathrm{Pl}+\mathrm{Qz}+\mathrm{Ilm}$, but in calcium-silicate hornfelses, sample 62-12: $\mathrm{MgHbl}_{0.56-0.66}+\mathrm{Aug}_{0.49-0.53}+$ $\mathrm{Bt}_{0.50}+\mathrm{Czo}\left(\mathrm{FeO} 6.65\right.$ wt.\%) $+\mathrm{Scp}+\mathrm{Grt}_{\text {Grs-Adr }}+\mathrm{Pl}_{0.36}$; $0.47 ; 0.54$ (Fig. 4).

In metapelites, andalusite and cordierite are represented by large porphyroblasts with numerous inclusions of fine (1-5 mm) flakes of biotite, quartz, graphite, opaque minerals and muscovite. Some $\mathrm{Mg}$ number differences in biotites and cordierites are apparently due to $\mathrm{Mg}$ number variations in original

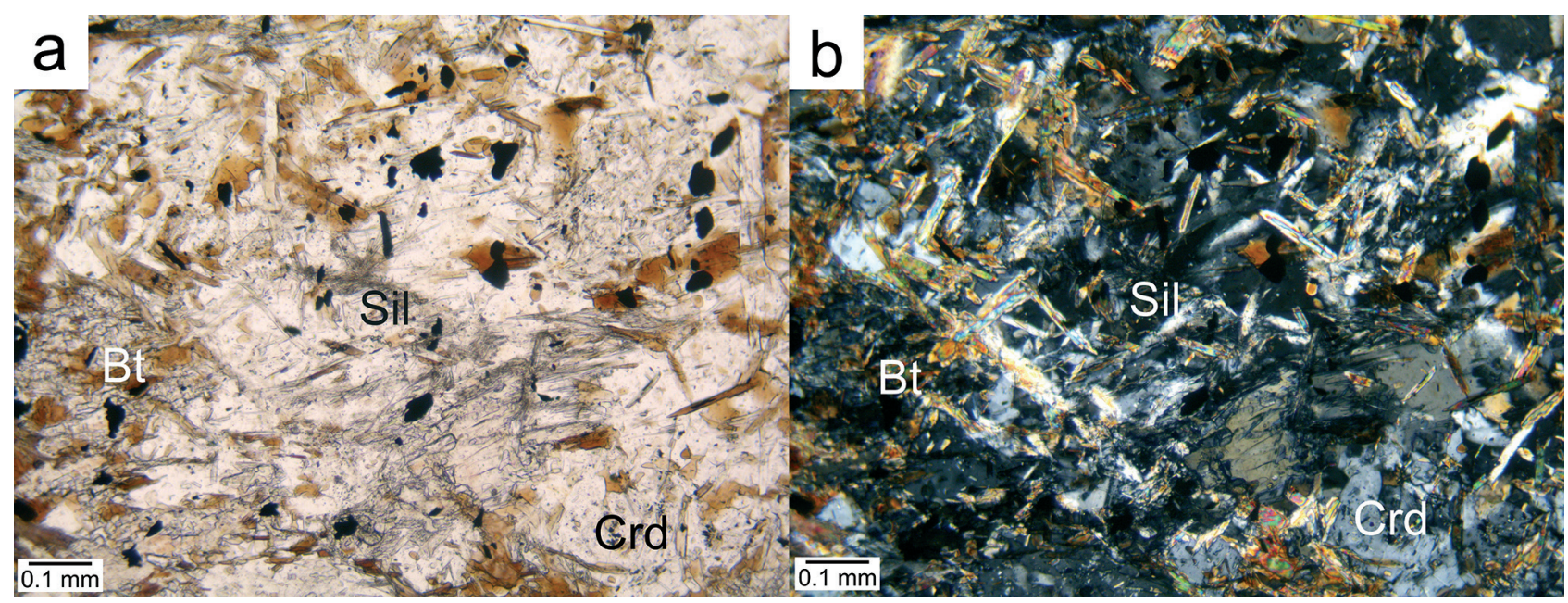

Fig. 3 Newly formed fibrolites after biotite: $a-$ PPL and b-XPL 


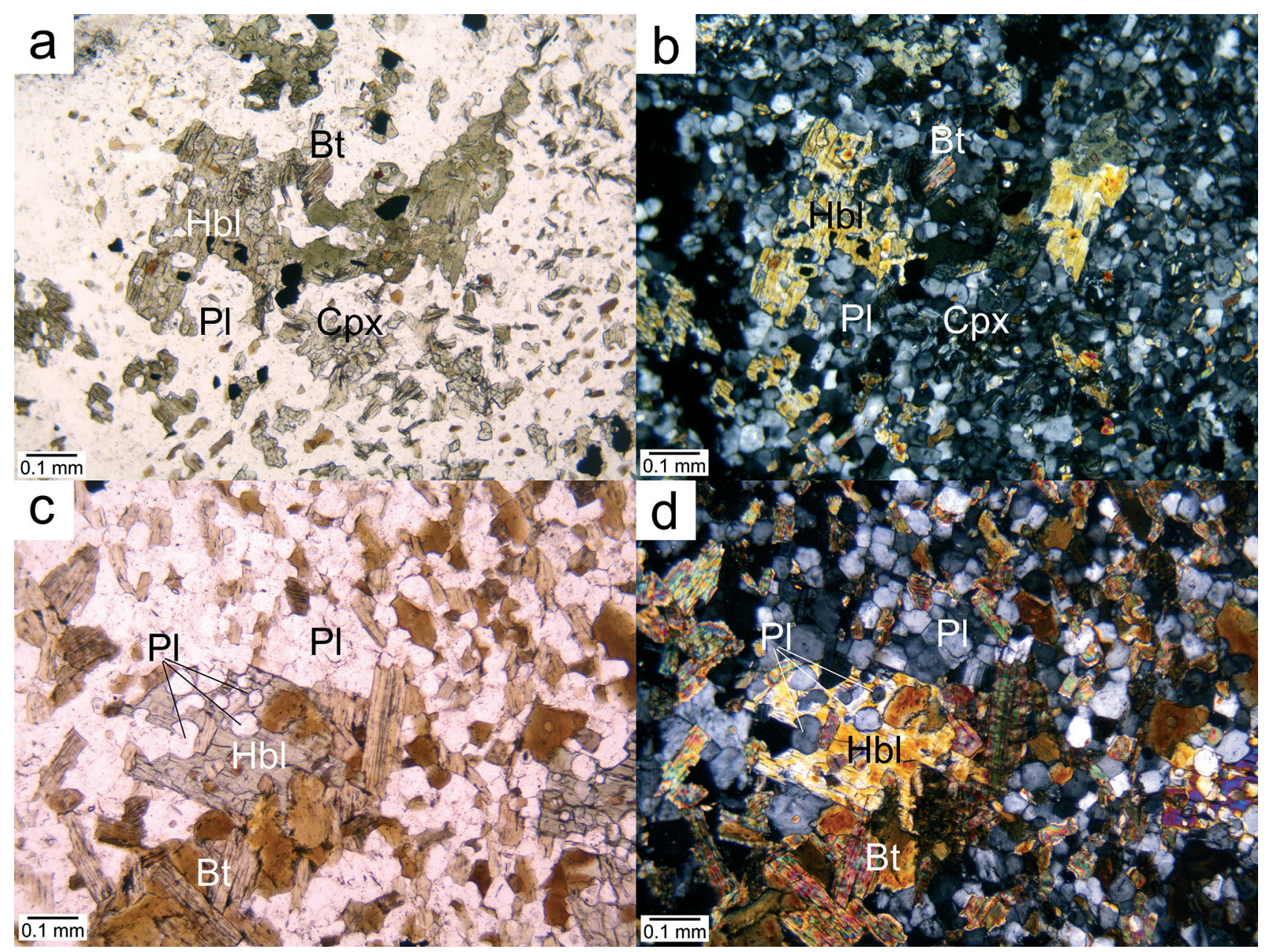

Fig. 4 Equilibrium mineral parageneses in the hornfels of the contact metamorphism zone III: a, c- PPL and b, d - XPL

rocks. Cordierite is one of the most widespread minerals in high-temperature associations of the Dizi Series hornfels. In some places, its amount exceeds $50 \%$ of the rock volume. Cordierite reveals polysynthetic and sectorial twinning. Helicitic texture, caused by the formation of cordierite after crenulation, is rarely observed (Fig. 5). Andalusite is also a common mineral in these rocks; it forms rare porphyroblasts, reaching up to several millimeters in size. Biotite forms small equant laths with the $\mathrm{Mg}$ number ranging from 0.36 to 0.50 . $\mathrm{TiO}_{2}$ content in biotite from metapelites and Carich rocks varies within a wide range. Muscovite, in contrast to biotite, is found in much smaller quantities. The phengite component in high-temperature muscovite is lower than in muscovite from the low-temperature aureole of the contact metamorphism. Mineral associations in xenoliths found in syenite-diorite are $\mathrm{Act}_{0.70-0.71}+\mathrm{Cum}_{0.65}+\mathrm{Bt}_{0.65}+\mathrm{Pl}_{0.49} \pm \mathrm{Qz}$ (sample 11$15-2$ ) and $\mathrm{MgHbl}_{0.66}+\mathrm{Cum}_{0.62-0.65}+\mathrm{Bt}_{0.64} \pm \mathrm{Pl}$ (sample 11-15-3). Cummingtonite is observed in association with magnesian hornblende or actinolite, biotite and calcic plagioclase (Fig. 6). Green hornblende poikiloblasts are abundant at sites of direct contact between xenoliths and intrusive rocks (Supplement 6). Actino- lite is a rare mineral in these rocks and is found only in association with cummingtonite and calcic plagioclase. It reveals prograde zoning: $\mathrm{Al}$ content and the $\mathrm{X}_{\mathrm{Mg}}$ value (0.72-0.75) increase from the center to the edges of the grains. The high $\mathrm{TiO}_{2}$ content in biotite from $\mathrm{CaO}$-rich rocks (Supplement 2, samples 62-12; $11-15-2$ ) is especially noteworthy. In general, it is significantly higher than in biotite from metapelites. The plagioclase composition varies from anorthite to labradorite inclusive; zoning is not observed. Opaque minerals are pyrrhotite and pentlandite. To determine the temperature of contact metamorphism in this zone, the following geothermometers were applied: Hbl-Cpx and Bt-Cpx (Perchuk, Ryabchikov 1976), Crd-Bt (Lavrentyeva, Perchuk 1981) and Ti in Ms (Chun-Ming $\mathrm{Wu}$, Hong-Xu Chen 2015). The temperature in this zone varies from 526 to $585^{\circ} \mathrm{C}$. The temperature of $620^{\circ} \mathrm{C}$ was estimated for xenolith in the largest Kirari intrusion using the Hbl-Pl thermometer (Spear 1981).

To design a scheme of facies and subfacies of metamorphism of the Dizi Series rocks, the authors used the scheme of metamorphic facies developed by S.P. Korikovsky (Korikovsky 1979, 1991; Korikovsky et al., 2018) (Fig. 7). 


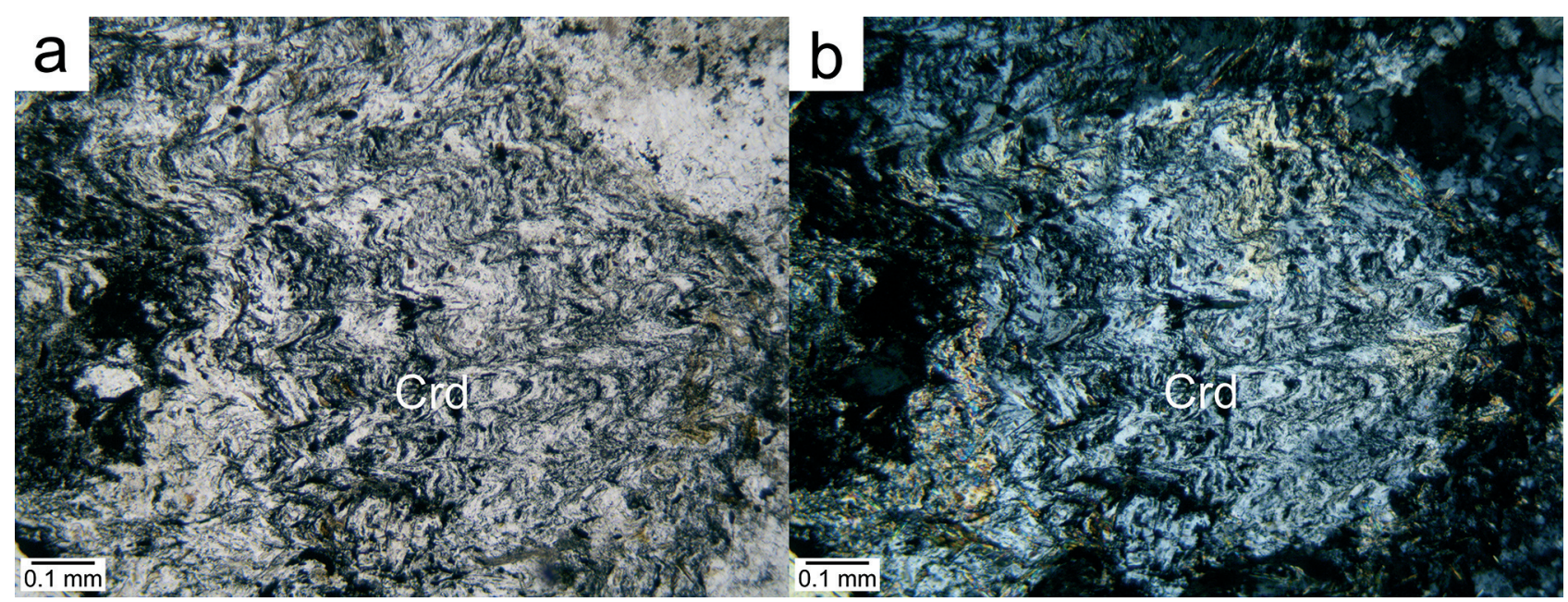

Fig. 5 Helicitic texture indicating cordierite formation after crenulation: $a-P P L$ and $b-X P L$

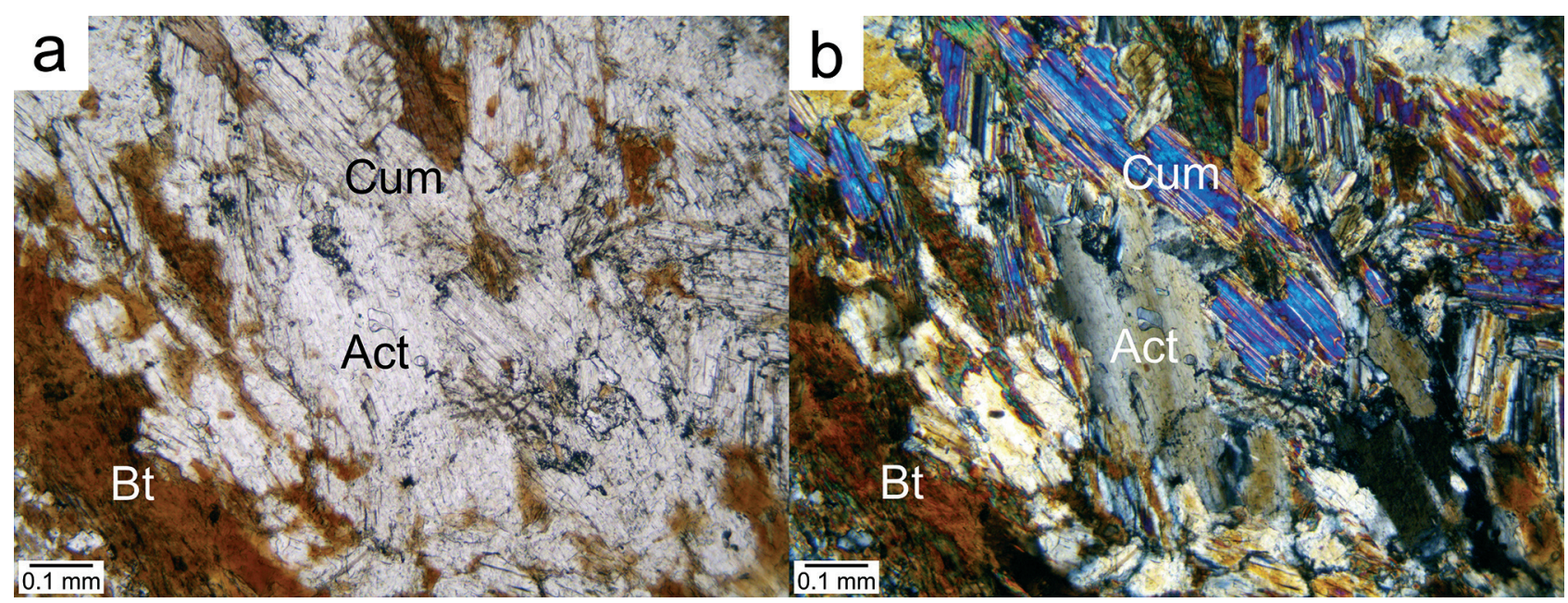

Fig. 6 Cummingtonite in association with actinolite, biotite and basic plagioclase: $a-P P L$ and $b-X P L$

\section{Results of isotope-geochronological studies}

In the studied sample of diorite porphyrite, zircons are mainly rounded and thus crystallographic forms cannot be determined (Fig. 8). There are also a few long-prismatic crystals with poorly developed bipyramidal facets (111) and clear asymmetric edges with an aspect ratio of 2.5-3, or prismatic-bipyramidal crystals with well-developed bipyramidal faces (311) and with an aspect ratio of 2-2.5, in which the weakly expressed zoning and a tendency to rounding of the crystal's outer contour are observed.

The U-Pb LA-ICP-MS dating (Table 2) allowed establishing three age populations of zircons in the studied sample: Zrn1 - ca. $2200 \mathrm{Ma}, \mathrm{Zrn} 2-458 \pm 29$ $\mathrm{Ma}$, and Zrn3 - 166.5 \pm 4.6 Ma. The first two populations are represented by rounded grains, while the Zrn3 population comprises crystals that have partly preserved their crystallographic forms. The analyses were carried out in central and marginal parts of zircon crystals. In all cases, the analyses conducted in marginal parts of the crystals revealed slightly younger ages than those determined when analyzing their central parts, indicating some $\mathrm{Pb}$ loss due to diffusion. However, all such pairs belong to the same age populations. We did not reveal any young overgrowths at the older cores.

The Zrn1 population is represented by a single grain, whose analysis yielded two nearly concordant results. The second (Zrn2) population represented by six grains was the most abundant. Analysis of these grains revealed concordant and discordant ages ranging from $362 \pm 18 \mathrm{Ma}$ to $495 \pm 11 \mathrm{Ma}\left({ }^{206} \mathrm{~Pb} /{ }^{238} \mathrm{U}\right.$ ages) due to the variable degree of $\mathrm{Pb}$ loss. The upper intercept age calculated for this population is 458 $\pm 29 \mathrm{Ma}$. Considering large variations in ages, it is unclear if the obtained upper intercept corresponds to a single igneous age of the protolith, or to a group of close ages of the rocks that were present in the source region. Finally, these zircons may be of detrital origin (as follows from their rounded shapes) and may be captured either from the host rocks during the emplacement or from the source region.

The third population (Zrn3) is represented by two 


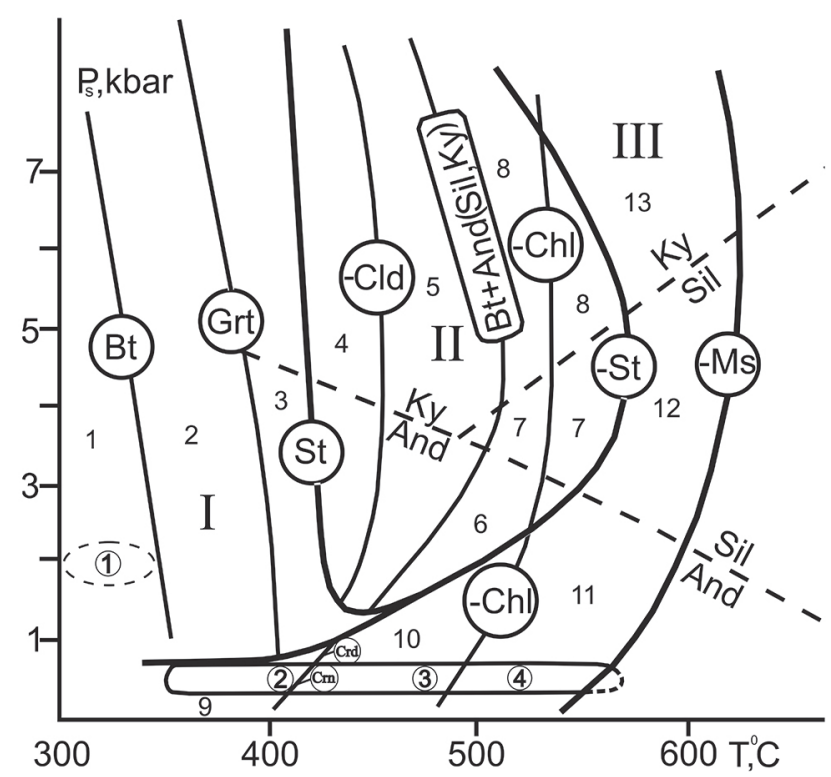

Fig. 7 Fields of regionally- and contact- metamorphosed rocks of the Dizi Series in the $\mathrm{P}_{\mathrm{s}} \mathrm{T}$ diagram of low and moderate temperature facies and subfacies of metamorphism (after Korikovsky 1979, with the additions by the authors). Roman figures indicate facies of regional metamorphism: I - greenschist, II - staurolite (epidote-amphibolite) and III - biotite-muscovite gneisses; Arabic figures indicate the subfacies of regional metamorphism (1-8) and those of contact metamorphism (9-13): 1 - chloritesericite, 2 - biotite, 3 - garnet, 4 - staurolite-chloritoid, 5 - staurolite-chlorite, 6 - staurolite-biotite-andalusite, 7 staurolite-biotite-sillimanite, 8 - staurolite-biotite-kyanite, 9 - albite-epidote-hornfels, 10 - andalusite-biotite-muscovite-chlorite-hornfels, 11 - andalusite-biotite-muscovite-hornfels, 12 - sillimanite-biotite-muscovite-hornfels, 13 - kyanite-biotite-muscovite-hornfels. Figures in circles indicate position of the regionally metamorphosed Dizi Series rocks -1 , and that of the Dizi Series rocks of contact metamorphism zones: I - 2, II -3 , and III -4 . Symbols of minerals indicate the appearance or disappearance (-) of minerals critical for each facies or subfacies
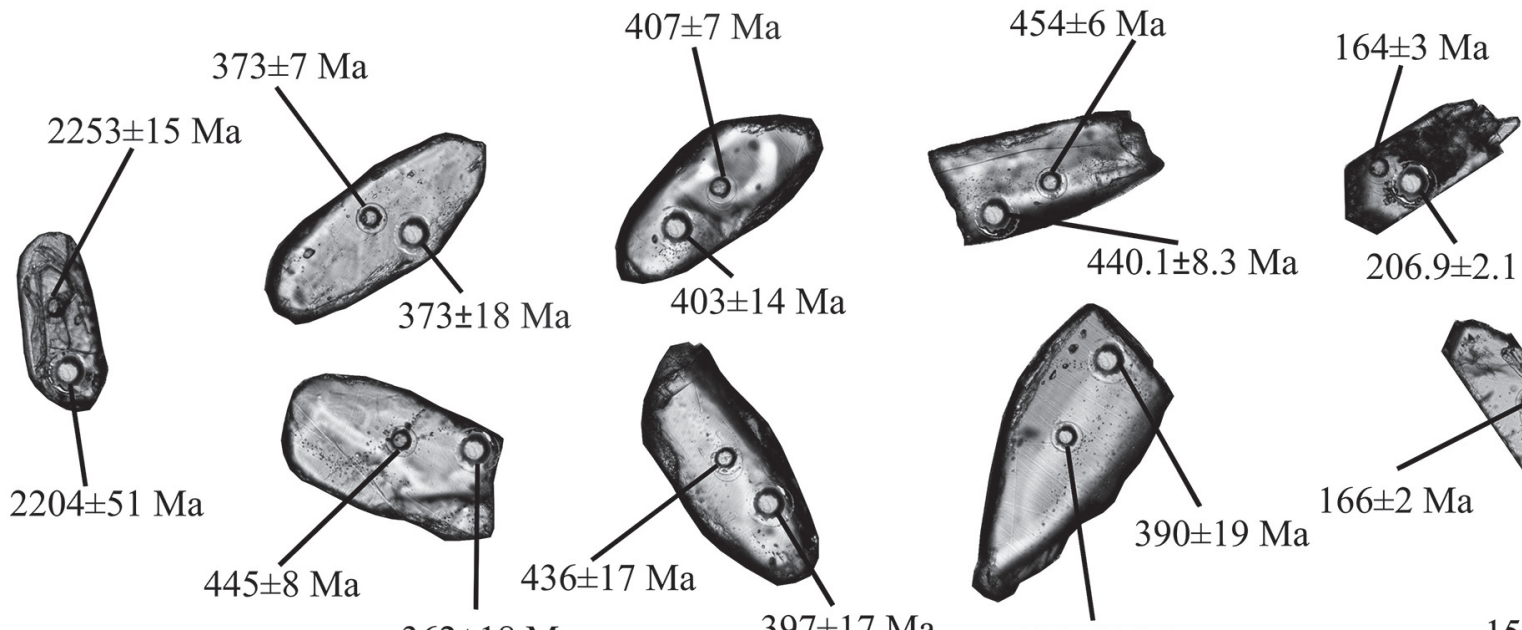

$403 \pm 14 \mathrm{Ma}$
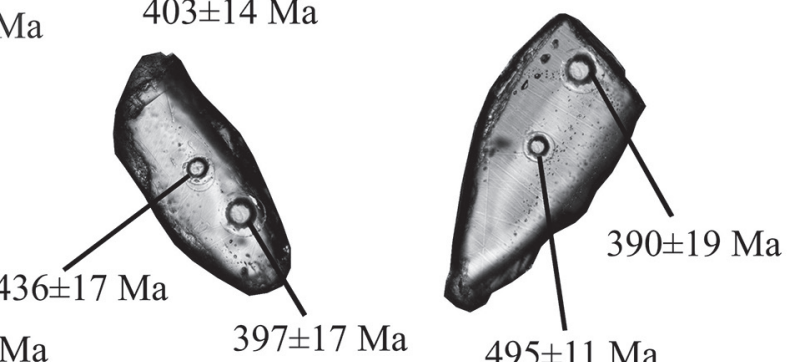

$495 \pm 11 \mathrm{Ma}$

Fig. 8 Optical images of dated zircons from the diorite-porphyritic dyke in the Dizi Series rocks 
Table 3 Hafnium isotope composition of zircon crystals from diorite-porphyritic dyke in the rocks of the Dizi Series

\begin{tabular}{|c|c|c|c|c|c|c|c|c|c|}
\hline Spot \# & $\begin{array}{c}{ }^{207} \mathrm{~Pb} /{ }^{206} \mathrm{~Pb} \\
\mathrm{age}, \mathrm{Ma}\end{array}$ & $\pm 1 \sigma$ & ${ }^{176} \mathrm{Lu} /{ }^{177} \mathrm{Hf}$ & ${ }^{176} \mathrm{Yb} /{ }^{177} \mathrm{Hf}$ & ${ }^{176} \mathrm{Hf} /{ }^{177} \mathrm{Hf}$ & $\pm 1 \sigma$ & ${ }^{176} \mathrm{Hf}^{177} \mathrm{Hf}_{\mathrm{T}}$ & $\varepsilon \mathrm{Hf}_{\mathrm{T}}$ & $\pm 2 \sigma$ \\
\hline DZ-14-3a & 440.1 & 8.3 & 0.002410 & 0.10050 & 0.282460 & 0.000043 & 0.282440 & -2.4 & 3.0 \\
\hline $\mathrm{DZ}-14-4 \mathrm{a}$ & 373 & 18 & 0.000608 & 0.02420 & 0.282402 & 0.000048 & 0.282398 & -5.4 & 3.4 \\
\hline $\mathrm{DZ}-14-5 \mathrm{a}$ & 2204 & 51 & 0.000830 & 0.03200 & 0.281195 & 0.000047 & 0.281160 & -7.6 & 3.3 \\
\hline DZ-14-6a & 158.6 & 3 & 0.009600 & 0.37830 & 0.283224 & 0.000079 & 0.283196 & 18.0 & 5.6 \\
\hline DZ-14-7a & 206.9 & 2.1 & 0.005516 & 0.21420 & 0.282843 & 0.000058 & 0.282822 & 5.9 & 4.1 \\
\hline DZ-14-8a & 403 & 14 & 0.001076 & 0.04430 & 0.282426 & 0.000042 & 0.282418 & -4.0 & 3.0 \\
\hline DZ-14-9a & 397 & 17 & 0.000247 & 0.00982 & 0.282413 & 0.000031 & 0.282411 & -4.4 & 2.2 \\
\hline DZ-14-12a & 362 & 18 & 0.000438 & 0.01690 & 0.282409 & 0.000041 & 0.282406 & -5.3 & 2.9 \\
\hline DZ-14-14a & 390 & 19 & 0.000627 & 0.02470 & 0.282368 & 0.000034 & 0.282363 & -6.2 & 2.4 \\
\hline
\end{tabular}
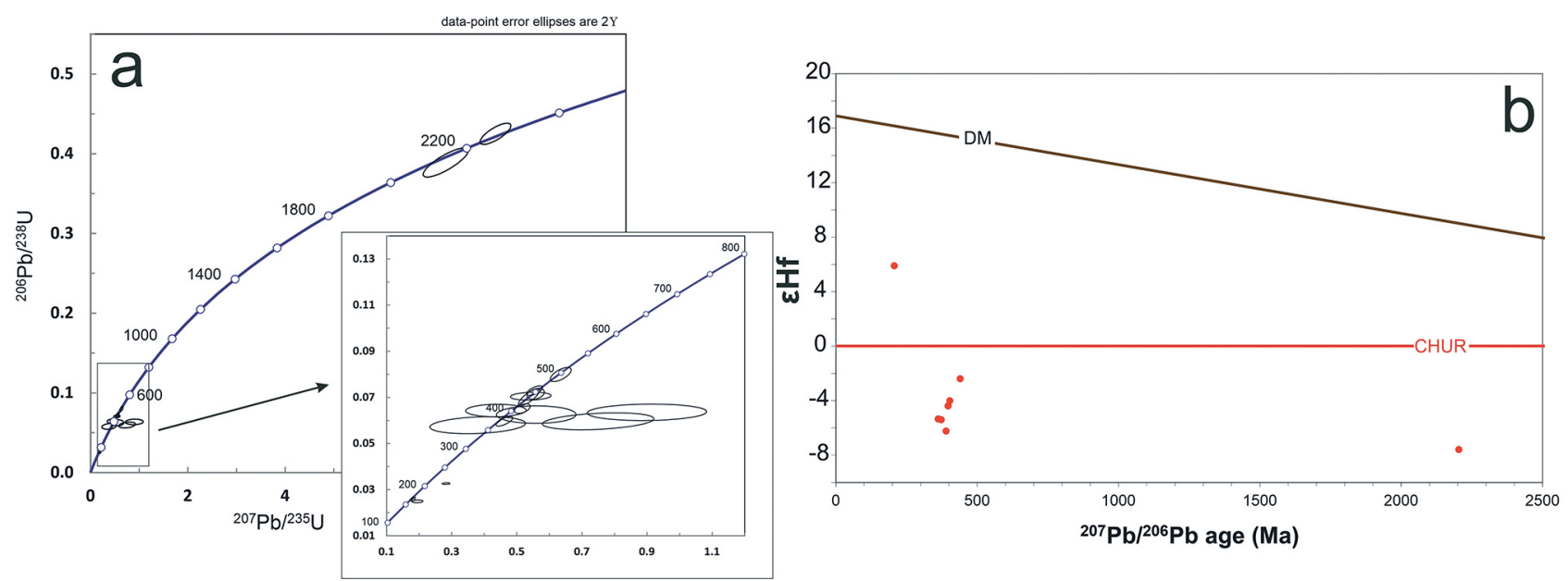

Fig. 9 U-Pb ages (a) and $\varepsilon H f$ values (b) in zircons from the diorite-porphyritic dyke in the Dizi Series rocks

Table 4 Concentrations of REE for zircons from diorite-porphiryte dyke in the Dizi Series

\begin{tabular}{|c|c|c|c|c|c|c|c|c|c|c|c|c|c|c|c|c|c|}
\hline Source file & $\begin{array}{c}{ }^{207} \mathrm{~Pb} /{ }^{206} \mathrm{~Pb} \\
\mathrm{age}, \mathrm{Ma}\end{array}$ & $\mathrm{Y}$ & $\mathrm{Nb}$ & $\mathrm{La}$ & $\mathrm{Ce}$ & $\mathrm{Pr}$ & $\mathrm{Nd}$ & $\mathrm{Sm}$ & $\mathrm{Eu}$ & $\mathrm{Gd}$ & $\mathrm{Dy}$ & $\mathrm{Yb}$ & $\mathrm{Lu}$ & $\mathrm{Hf}$ & $\mathrm{Th}$ & $\mathrm{U}$ & $\mathrm{Pb}$ \\
\hline $\mathrm{DZ}-14-3$ & 440 & 3120 & 1.88 & $\mathrm{BDL}$ & 9.73 & 1.37 & 15.2 & 15.5 & 0.61 & 89.5 & 323 & 728 & 123.9 & 8619 & 470 & 366 & 99.6 \\
\hline DZ-14-4 & 373 & 623 & 1.23 & $\mathrm{BDL}$ & 5.28 & 0.39 & 2.59 & 3.15 & 0.29 & 14.4 & 59.4 & 184 & 33.9 & 9700 & 77 & 103 & 20.2 \\
\hline $\mathrm{DZ}-14-5$ & 2204 & 856 & 3.55 & $\mathrm{BDL}$ & 357 & 114 & 288 & 29.7 & 4.63 & 37.3 & 87.5 & 231 & 42.7 & 8630 & 142 & 331 & 209 \\
\hline DZ-14-6 & 159 & 5707 & 9.16 & $\mathrm{BDL}$ & 302 & 9.8 & 74 & 50.3 & 20.6 & 178 & 579 & 1883 & 338.6 & 6333 & 3703 & 1668 & 291 \\
\hline DZ-14-7 & 207 & 4790 & 33.3 & $\mathrm{BDL}$ & 661 & 23.9 & 135.8 & 61.9 & 18.9 & 171.5 & 446 & 1326 & 233 & 7452 & 34610 & 7730 & 2670 \\
\hline DZ-14-8 & 403 & 1303 & 1.3 & $\mathrm{BDL}$ & 5.24 & 0.31 & 5.7 & 7.1 & 0.45 & 33.7 & 132.3 & 321 & 54 & 8661 & 179.9 & 173.6 & 37.4 \\
\hline DZ-14-9 & 397 & 286 & 1.01 & $\mathrm{BDL}$ & 3.55 & 0.21 & 1.07 & 1.63 & 0.247 & 6.8 & 27.6 & 75.7 & 13.46 & 9646 & 37.7 & 60.4 & 13.8 \\
\hline DZ-14-12 & 362 & 457 & 1.12 & $\mathrm{BDL}$ & 4 & 0.26 & 1.44 & 2.22 & 0.3 & 8.7 & 39.1 & 135 & 24.2 & 9679 & 48.4 & 79.2 & 9.3 \\
\hline DZ-14-14 & 390 & 717 & 1.25 & $\mathrm{BDL}$ & 5.01 & 0.53 & 1.86 & 3.72 & 0.185 & 16.7 & 65.2 & 196 & 34.8 & 9449 & 82.6 & 114.3 & 18 \\
\hline
\end{tabular}

Note: $\mathrm{BDL}=$ below detection limit.

ries indicate that they formed under conditions of the chlorite-sericite subfacies of greenschist facies. The paragenesis of Act $(\rightarrow \mathrm{MgHbl})_{0.60: 0.62}+\mathrm{Pmp}_{0.53}+\mathrm{Phr}+$ $\mathrm{Ab}+\mathrm{Qz}$ recorded in a single case in the graywacke rock is indicative of the prehnite-pumpellyite facies. Apparently, relics of this facies were locally preserved in the Dizi Series. Due to the complexity of the relief and inaccessibility of the outcrops, it was not possible to find other occurrences of the rocks of the prehnitepumpellyite facies, although the possible existence of weakly metamorphosed or completely unmetamorphosed rocks was admitted. It is noteworthy that previously the prehnite-pumpellyite association was not established in the Greater Caucasus. According to the mineral assemblages of regional metamorphism, and data of low-temperature Chl-geothermometry, the regional metamorphism of the Dizi Series took place under conditions of the chlorite-sericite subfacies of the greenschist facies $\left(\mathrm{T}=300-350^{\circ} \mathrm{C}\right.$ and $\mathrm{P}=1.5-$ 2.3 kbar; Fig. 7). 


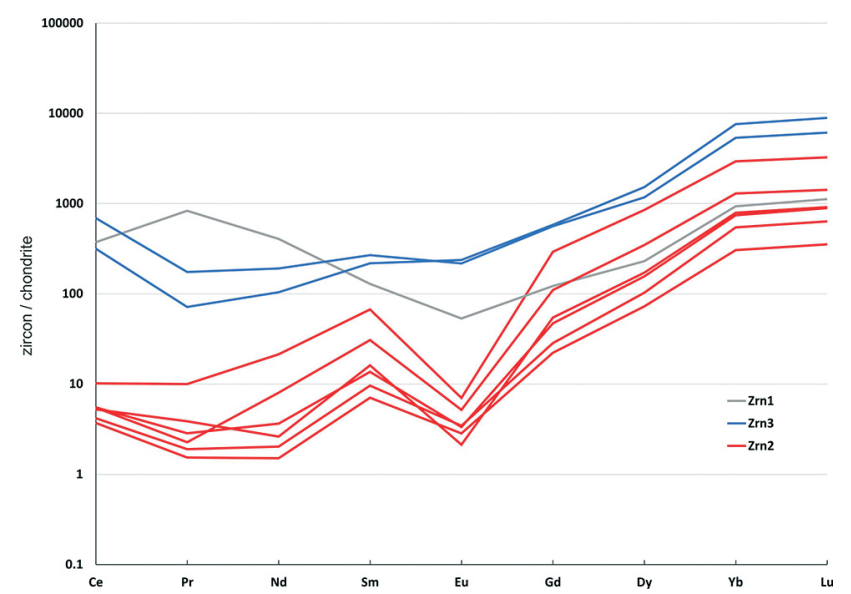

Fig. 10 The chondrite-normalized pattern of REE for zircons from the diorite-porphyritic dyke in the Dizi Series

Based on the mineral assemblages, three zones of contact metamorphism can be distinguished. The lowest-temperature (external) zone (I) is characterized by completely preserved primary structural and textural features. According to the characteristic mineral associations, rocks of zone I belong to the albiteepidote-hornfels subfacies. The thickness of this zone varies from 50 to $150 \mathrm{~m}$. PT conditions of its formation were defined on the basis of mineral assemblages alone $\left(\mathrm{T}=350-435^{\circ} \mathrm{C}\right.$ and $\left.\mathrm{P}=0.3-0.8 \mathrm{kbar}\right)$, as there were no electron microprobe analysis results. The absence of garnet in metapelites confirmed low pressure in the contact aureole.

The second zone (II), which lies next to zone I, is also in direct contact with thin intrusive bodies. As the temperature rises, the degree of rock recrystallization increases and, therefore, relics of primary minerals disappear, although their structural features are still preserved locally. Garnet is not found in metapelites of zone II either, the chlorite + quartz + magnetite association occurs instead. The presence of equilibrium chlorite in the mineral paragenesis of the porphyroblastic schist of zone II is indicative of the early stage of contact metamorphism, when potassium feldspar in association with andalusite has not appeared yet and chlorite stability persists. Mineral assemblages of zone II correspond to the andalusite-biotite-muscovite-chlorite-hornfels subfacies of the staurolite (epidote-amphibolite) facies. The thickness of this zone is $40-150 \mathrm{~m}$. According to its mineral association and data of geothermometers, the P-T conditions of zone II formation are $410-495^{\circ} \mathrm{C}$ and $0.3-0.8$ kbar.

The third zone (III) is characterized by the highest temperature. It develops in direct contact with intrusions, and in xenoliths. Its width reaches $10 \mathrm{~m}$. In the high-temperature part of the zone, fibrolite is formed in metapelites. In the mineral parageneses of this zone, as a rule, And $+\mathrm{Kfs}$ is present only in association with $\mathrm{Ms}$, and the Sil + Kfs association has never been observed. The mineral associations of zone III indicate that they are mainly in the stability field of andalusite and muscovite with quartz, but near the line of the polymorphic transformation - andalusite $\rightarrow$ sillimanite (replacement of biotite by fibrolite is sporadically observed). Conditions of contact metamorphism of zone III fully correspond to the andalusite-biotitemuscovite-hornfels subfacies of the biotite-muscovite gneiss facies. These are the most common conditions for the majority of the mid-temperature interval of contact aureoles (Reverdatto 1970; Korikovsky 1979). The sporadic development of fibrolite after biotite indicates that the high-temperature part of the zone directly borders the biotite-sillimanite-potassium feldspar facies, and the low-temperature part is adjacent to the andalusite-biotite-muscovite-chlorite subfacies of the staurolite facies. According to mineral associations and geothermometers, P-T conditions of formation of rocks in zone III are $490-570^{\circ} \mathrm{C}$ and $0.3-0.8$ kbar.

Thus, the contact metamorphism of the Dizi Series rocks proceeded under the conditions of albite-epidote-hornfels, andalusite-biotite-muscovite-chloritehornfels and andalusite-biotite-muscovite-hornfels subfacies at the maximum temperature of ca. $570^{\circ} \mathrm{C}$ and pressure of ca. $0.3-0.8 \mathrm{kbar}$. It occurred at a significantly higher temperature and lower pressure than the preceding regional metamorphism $(\mathrm{T}=300$ $350^{\circ} \mathrm{C}$ and $\left.\mathrm{P}=1.5-2.3 \mathrm{kbar}\right)$.

All the established transformations of minerals in regionally and contact-metamorphosed rocks are summarized in Table 1 in the order of increasing temperature. Based on the mineral assemblages characteristic of regional and contact metamorphism of all rock types of the Dizi Series, the following scheme of metamorphic facies and subfacies can be suggested (Fig. 7).

As mentioned above, the U-Pb LA-ICP-MS zircon dating (Table 2) of the diorite-porphyritic intrusion yielded three zircon age populations: Zrn1 - ca. $2200 \mathrm{Ma}, \mathrm{Zrn} 2-458 \pm 29 \mathrm{Ma}$, and Zrn3 $-166.5 \pm 4.6$ Ma. The first two populations were captured by the diorite magma from the ancient crystalline basement. The simultaneous analysis of the Hf isotope composition and trace elements in zircons allows resolving their origin-related issues. The low $\varepsilon \mathrm{Hf}$ value of -7.6 in zircon of the oldest population allows linking the origin of this zircon source rocks to the remelting of the Archean precursor. Interestingly, according to the maps of the East-European platform, the Greater Caucasus occurs on the very margin of the Sarmatian segment of the platform (e.g., Bogdanova et al. 2016). However, zircons with such low $\varepsilon$ Hf values are not typical of Sarmatia, in the early Paleoproterozoic time, it was affected by very intense juvenile magmatism (e.g, Shumlyanskyy et al. 2018). According to the chemical composition, this zircon can be 
classified as derived from rocks of alkaline affinity syenites or lamproites (Belousova et al. 2002).

Zircons of the second population (Zrn2) also show negative $\varepsilon H f$ values (-6.2 to -2.4$)$, indicative of the Paleoproterozoic to Mesoproterozoic crustal source for the melt from which these zircons have crystallized. However, the composition of this melt remains unclear. The pattern of REE in these zircons corresponds to zircons from pegmatites in mafic rocks (Shumlyanskyy et al. 2017). Low Y, Th and U concentrations are also indicative of the mafic composition of the melt (Belousova et al. 2002).

The youngest population of zircons (Zrn3) that supposedly crystallized from the diorite melt has high $\varepsilon \mathrm{Hf}$ values, indicative of the juvenile origin of the melt, i.e., it was derived due to the melting of the mantle source rather than the older crustal precursor. The chemical composition of zircons supports their crystallization from the intermediate to felsic melt, whereas rather weakly fractionated REE patterns indicate a significant role of fluids as such a pattern is more common to "hydrothermal" zircons.

It should be noted that the ages of the crystalline basement of the Greater Caucasus and the Georgian Block bordering it from the south, which were determined employing the SHRIMP and LA-ICP-MS methods (Gerasimov et al. 2010; Somin 2011; Gamkrelidze et al. 2020), are similar to Zrn1 and Zrn2 populations. The age of crystallization of diorite-porphyrite (Zrn3, $166.5 \pm 4.6 \mathrm{Ma}$ ) is fully consistent with the age of the Chegem orogeny. The ages of the Dizi Series intrusions (176-165 Ma) determined using the K-Ar method by Dudauri and Togonidze (2016) are also similar.

The authors of this paper compared the Dizi series with synchronous deposits in the adjacent regions. The Paleozoic rocks similar to those of the Dizi Series (chlorite-, prehnite-, actinolite-, clayey-carbonaceous and sericite-carbonaceous schists, etc.; Savitsina, Plakhotny 1965; Chernyak 1969) occur under the Cretaceous and Jurassic deposits within the folded basement of the Crimean Plain where they were disclosed by the wells. Most of these rocks have undergone low-grade metamorphism and, to a lesser extent, deep epigenesis (Novikov 1963; Savitsina, Plakhotny 1965; Snegiryova, Shalya 1966). Some of the rocks resemble the Lower Carboniferous Carapelite formation of the southern Dobrudgea (Chernyak 1969).

Interestingly, the folded basement of the Crimea hosts numerous Middle Jurassic intrusions of gabbro-diabases, diabases, diabase-porphyrites, diorites, quartz-diorites, syenites, granite-porphyrites (Chernyak 1969; Meijers 2010; Shniukova 2016, Shumlyanskyy, Shumlyanskyy 2019). Intrusions of similar age and composition are also widely distributed in the Dizi Series. The widespread hornfelses, muscovite-quartzcordierite and cordierite-quartz-feldspar mica schists
(Chernyak 1969), apparently similar to those found in the Dizi Series, were formed as a result of the contact metamorphism of the basement rocks in the Crimea.

Some authors suggest that the Dizi Series represents part of the Triassic Upper Karakaya Complex that includes the Karakaya and Küre complexes in the Sakarya Zone in Turkey and the Tauric flysch in the Crimea (Okay et al. 2015; Okay, Topuz 2016). According to these authors, the Upper Karakaya Complex shares several common features with the Akgöl Formation of the Central Pontides, including tectonic setting, lithology and age, and was most probably deposited in the same forearc on the southern margin of Laurasia. They assume that the Tauric flysch in the Crimea and the Dizi Series in the Caucasus likely represent parts of the same basin. They include exotic Carboniferous and Permian shallow marine limestone blocks (Leven, Okay 1996), most likely derived from Gondwana, and blocks of Devonian, Carboniferous and Permian radiolarian cherts (Okay et al. 2011a), representing fragments of the Paleo-Tethyan oceanic crust.

The Middle Jurassic epoch in the northern part of the Mediterranean belt, including the Caucasus, is marked by significant tectonic-magmatic activization, whereas in the Dizi Series, only magmatism was intensely manifested. Before the complete cessation of volcanic activity, at the end of the Bajocian age, tectonic movements intensified reaching their maximum in the Bathonian time and persisted thereafter throughout the Bathonian time. The mode of subsidence, extension and destruction of the crust, which existed in the Bajocian, was accompanied by extensive volcanism, and at the end of the Bajocian, by gabbroic magmatism. After this regime had been replaced by compression and uplift in the Bathonian time, the felsic melt produced during the fusion of the crystalline substrate was expelled towards the surface. At the end of the Bajocian, gabbroic melts intruded rocks of the Dizi Series that were intensely deformed during the Early Cimmerian orogeny, and later on, in the Bathonian, felsic melts emplaced into the rocks of the series. In spite of the fact that intense tectonic movements in the Caucasus continued until the end of the Bathonian time, intrusive massifs did not experience any significant deformations, except for rupture dislocations.

\section{CONCLUSIONS}

Based on geological, petrological, mineralogical and geochronological studies of the Dizi Series rocks, the following conclusions can be drawn.

Rocks of the Dizi Series underwent regional metamorphism under conditions of the chlorite-sericite subfacies of the greenschist facies at the temperature of $300-350^{\circ} \mathrm{C}$ and pressure of $1.5-2.3 \mathrm{kbar}$. In a sin- 
gle case, in the graywacke rock, we recorded a relict paragenesis of the prehnite-pumpellyite facies: Act $_{0.59}$ $+\mathrm{Pmp}_{0.53}+\mathrm{Phr}+\mathrm{Ab}+\mathrm{Qz}$.

Emplacement of the Middle Jurassic igneous rocks into the Dizi Series caused contact metamorphism zonation. Three zones of contact metamorphism were identified. Progressive variations in the composition and characteristic features of minerals were established. The appearance and disappearance of minerals in contact metamorphosed rocks define internal and external boundaries between contact metamorphism zones.

The following subfacies of contact metamorphism were established: albite-epidote-hornfels, andalusitebiotite-muscovite-chlorite-hornfels, and andalusitebiotite-muscovite-hornfels. P-T conditions of metamorphism were determined for each subfacies based on the geothermometer readings, mineral assemblages and nature of transformations in the mineral composition. Contact metamorphism of the Dizi Series took place at the maximum temperature of ca. $570^{\circ} \mathrm{C}$ and pressure of ca. 0.3-0.8 kbar. It occurred at a significantly higher temperature and lower pressure than the preceding regional metamorphism.

Based on the data of U-Pb LA-ICP-MS zircon dating, three age populations were distinguished in the diorite-porphyrite intrusion: Zrn1 (ca. $2200 \mathrm{Ma}$ ) and Zrn2 (458 $\pm 29 \mathrm{Ma})$ represent xenocrysts and/or detrital grains, which were captured by diorite-porphyrite magma from the ancient crystalline basement, and Zrn3 (166.5 $\pm 4.6 \mathrm{Ma})$ formed during the crystallization of diorite-porphyrite magma. The age of $\mathrm{Zrn} 3$ is consistent with that of the Chegem orogeny.

Based on rock associations, nature of regional and contact metamorphism, age of bedrocks and magmatism, as well as spatial location of the Dizi Series and the folded system of the Crimean Plain, we suggest that they developed simultaneously in the same environment and in a similar geological setting.

The Middle Jurassic epoch in the Caucasus is marked by significant magmatic activization. At the end of the Bajocian, gabbro melts intruded into the Dizi Series rocks, which had been intensely deformed during the Early Cimmerian orogeny. Later, in the Bathonian Age, felsic rocks were emplaced in the rocks of the Series. Although intense tectonic movements in the Caucasus continued until the end of the Bathonian, intrusions do not bear any signs of deformation, except for rupture dislocations.

\section{ACKNOWLEDGEMENTS}

This work was supported by the Shota Rustaveli National Science Foundation (SRNSF) [PHDF-19159, Regional and Contact Metamorphism of the Dizi Series]. The authors of the article express their deep gratitude to the late Professor S.P. Korikovsky for valuable recommendations during the work on the article and for support in carrying out analytical work on the microprobe at IGEM RAS. We acknowledge the Curtin Research Office for providing support to LS. The paper has benefitted from constructive comments from three anonymous reviewers. We also thank Mrs Laima Monkiene for the language revision.

\section{REFERENCES}

Adamia, Sh.A. 1968. Dojurskie obrazovanija Kavkaza [Pre-Jurassic Formations of the Caucasus]. Metsniereba, Tbilisi, 294 pp. [In Russian].

Belousova, E., Griffin, W.L., O'Reilly, S.Y., Fisher, N.L. 2002. Igneous zircon: trace element composition as an indicator of source rock type. Contributions to Mineralogy and Petrology 143, 602-622. https://doi.org/10.1007/s00410-002-0364-7

Belov, A.A. 1986. Paleozoj Kavkaza i problemy Paleotetisa [Paleozoic of the Caucasus and problem of Paleotethys] Geotektonika 3, 17-29. [In Russian].

Bogdanova, S.V., Gorbatschev, R., Garetsky, R.G. 2016. EUROPE - East European Craton, Reference Module in Earth Systems and Environmental Sciences, Elsevier. https://doi.org/10.1016/B978-0-12-409548-9.10020-X

Bouvier, A., Vervoort, J.D., Patchett, P.J. 2008. The Lu-Hf and Sm-Nd isotopic composition of CHUR: constraints from unequilibrated chondrites and implication for the bulk composition of terrestrial planets. Earth and Planetary Science Letters 273, 48-57. https://doi.org/10.1016/j.eps1.2008.06.010

Cathelineau, M. 1988. Cation site occupancy in chlorites and illites as a function of temperature. Clay Minerals 23, 471-485.

Cathelineau, M., Nieva, D. 1985. A chlorite solid solution geothermometer the Los Azufres (Mexico) geothermal system. Contributions to Mineralogy and Petrology 91, 235-244.

Chernyak, N.I. 1969. Dokembrij i paleozoj [Precambrian and Paleozoic]. In: Starikova, L.M., Shmakova, T.M. (eds). Geologija SSSR, VIII, Krym. Chast' I. Geologicheskoe opisanie [Geology of the USSR, VIII, Crimea. Part I. Geological description]. Nedra, Moskva, 66-74. [In Russian].

Chikhradze, G.A. 2005. O stratigrafii i litologii verhnepaleozojsko-triasovyh otlozhenij “Dizskoj serii” Bol'shogo Kavkaza [On the stratigraphy and lithology of the Upper Paleozoic-Triassic deposits of the "Dizi Series" of the Greater Caucasus]. Proceedings of Georgian Technical University 2 (456), 88-94. [In Russian].

Chu, N.-C., Taylor, R.N., Chavagnac, V., Nesbitt, R.W., Boella, R.M., Milton, J.A., German, C.R., Bayon, G., Burton, K. 2002. Hf isotope ratio analysis using multicollector inductively coupled plasma mass spectrometry: an evaluation of isobaric interference corrections. Journal of Analytical Atomic Spectrometry 17, 15671574. https://doi.org/10.1039/b206707b 
Chun-Ming, Wu., Hong-Xu, Chen. 2015. Calibration of a Ti-in-muscovite geothermometer for ilmenite- and $\mathrm{Al}_{2}$ $\mathrm{SiO}_{5}$-bearing metapelites. Lithos 212-215, 122-127. https://doi.org/10.1016/j.lithos.2014.11.008

Coombs, D.S., Nakamura, Y., Vuagnat M. 1976. Pumpellyite-actinolite facies schists of the Taveyanne Formation near Loeche, Valais, Switzerland. Journal of Petrology 17 (4), 440-471.

Dudauri, O., Togonidze, M. 2016. Petrologija i izotopnaja geohronologija Mezozojskih intruzivnyh kompleksov Gruzii [Petrology and Isotope Geochronology of Mesozoic Intrusive Complexes of Georgia]. Proceedings of Al. Janelidze Institute of Geology 128, 370 pp. [In Russian].

Gamkrelidze, I.P. 1989. Geodinamicheskaja evoljucija i mehanizm formirovanija struktury Sredizemnomorskogo skladchatogo pojasa [Geodynamic Evolution and Mechanism of Formation of the Structure of the Mediterranean Fold Belt]. In: Belov, A.A., Satian, M.A. (eds) Geodinamika Kavkaza [Geodinamics of the Caucasus]. Nauka, Moskva, 28-36. [In Russian].

Gamkrelidze, I., Shengelia, D. 2005. Dokembrijsko-paleozojskij regional'nyj metamorfizm, granitoidnyj magmatizm i geodinamika Kavkaza [Precambrian-Paleozoic regional metamorphism, granitoid magmatism and geodynamics of the Caucasus]. Nauchni Mir, Moskva, 458 pp. [In Russian].

Gamkrelidze, I., Shengelia, D., Chichinadze, G., Okrostsvaridze, A., Lee Y.-H., Beridze G., Vardanashvili K. 2020. U-Pb LA-ICP-MS dating of zoned zircons from the Greater Caucasus pre-Alpine crystalline basement: Evidence for Cadomian to Late Variscan evolution. Geologica Carpathica 71, 3, 249-263. https://doi.org/10.31577/GeolCarp.71.3.4

Gerasimov, V.I. Pismenni, A.N., Enna, N.L. 2010. Cirkonometrija metagranitoidov kristallinikuma Bol'shogo Kavkaza [Zirconometry of Meta-granitoids of the Crystallinicum of the Greater Caucasus]. In: Tezisy dokladov XI Vserossijskogo Petrograficheskogo Soveshhanija "Magmatizm $i$ Metamorfizm v Istorii Zemli" [Abstracts of the XI All-Russian Petrographic Conference "Magmatism and Metamorphism in the History of the Earth"], August 2010, Ekaterinburg, 167-168. [In Russian].

Goishvili, B.A. 1960. O zonal'nosti skarnov massiva g. Kirar v Verhnej Svaneti [About zonality of skarns from the Kirar massif in Upper Svaneti]. Proceedings of CIMR 11 (4) b, 45-67. [In Russian].

Goishvili, B.A. 1961. Effuzivnyj vulkanizm v Dizskoj svite [Effusive volcanism in the Dizi suite]. Proceedings of CIMR 3 (5), 60-68. [In Russian].

Hawthorne, F.C., Oberti, R., Harlow, G.E., Maresch, W.V., Martin, R.F., Schumacher, J.C., Welch, M.D. 2012. Nomenclature of the amphibole supergroup. American Mineralogist 97, 2031-2048. https://doi.org/10.2138/am.2012.4276

Hiller, S., Velde, B. 1991. Octahedral occupancy and the chemical composition of diagenetic (low-temperature) chlorites. Clay Minerals 26, 149-168.
Holland, T., Blundy, J. 1994. Non-ideal interactions in calcic amphiboles and their bearing on amphibole-plagioclase thermometry. Contributions to Mineralogy and Petrology 116, 433-447. https://doi.org/10.1007/BF00310910

Jackson, S.E., Pearson, N.J., Griffin, W.L., Belousova, E.A. 2004. The application of laser ablation-inductively coupled plasma-mass spectrometry to in situ U-Pb zircon geochronology. Chemical Geology 211, 47-69. https:// doi.org/10.1016/j.chemgeo.2004.06.017

Jaques, A.L., Blake, D.H., Donchak, P.J.T. 1982. Regional metamorphism in the Selwyn Range area, north-west Queensland. BMR Journal of Australian Geology and Geophysics 7, 3, 181-196.

Javakhishvili, I., Beridze, G. 2020. Dizis seriis (kavkasioni) bat'uri magmatitebis petrogeokimia [Petrogeochemistry of the Dizi Series (the Greater Caucasus) Bathonian magmatites]. Proceedings of Al. Janelidze Institute of Geology 132, 157-198. [In Georgian].

Javakhishvili, I., Tsutsunava, T., Shengelia, D., Chichinadze, G., Beridze, G. 2020. Regional and Contact Metamorphism of the Dizi Series (the Greater Caucasus). In: EGU General Assembly 2020, May 2020, Online, EGU2020-2952, https://doi.org/10.5194/egusphere-egu2020-2952

Jowett, E.C. 1991. Fitting iron and magnesium into the hydrothermal chlorite geothermometer. In: GAC/MAC/ SEG Joint Annual Meeting, May 1991, Toronto, Canada.

Kazmin, V.G., Sborshikov, I.M. 1989. Paleozojskie i rannekimmerijskie deformacii na Kavkaze $\mathrm{i}$ ih mesto $\mathrm{v}$ razvitii Tetisa [Paleozoic and Early Kimmerian deformation in the Caucasus and their place in evolution of Tethys]. In: Belov, A.A., Satian, M.A. (eds) Geodinamika Kavkaza [Geodinamics of the Caucasus]. Nauka, Moskva, 45-54. [In Russian].

Korikovsky, S.P. 1979. Facii metamorfizma metapelitov [Metamorphic facies of metapelites]. Nauka, Moskva, 263 pp. [In Russian].

Korikovsky, S.P. 1991. Principy podrazdelenija metamorficheskih subfacij v zonal'nyh oreolah [Principles of subdivision of metamorphic subfacies in zonal aureoles]. In: Korikovsky, S.P. (ed.) Petrologija metamorficheskih kompleksov Bol'shogo Kavkaza [Petrology of the Metamorphic Complexes of the Greater Caucasus]. Nauka, Moskva, 46-51. [In Russian].

Korikovsky, S.P., Tzambel, B., Miklosh, J., Putish, M. 1984. Metamorfizm kristallikuma malyh Karpat: jetapy, zonal'nost', svjaz' s granitoidami [Metamorphism of the crystallinikum of the Lesser Carpathians: stages, zonality, relationship with granitoids]. Geologicky zbornik, Geologica Carpathica 35, 4, 437-462. [In Russian].

Korikovsky, S.P., Yanak, M., Boronikhin, V.A. 1986. Geotermometrija i izmenenie mineral'nyh ravnovesij pri perekristallizacii granat-sljudjanyh slancev v kordieritovye rogoviki v oreole rohovskih granitov (Slovackoe rudogor'e, rajon ss. Rohovce - Hizhne) [Geothermom- 
etry and alteration of mineral equilibria during the recrystallization of garnet-mica schist to cordierite hornfelses in the aureole of Rokhovsky granites (Slovak Highlands, village of Rochovce - Chyžné)]. Geologicky zbornik, Geologica Carpathica, 37, 4, 607-633. [In Russian].

Korikovsky, S.P., Shengelia, D.M., Somin, M.L. 1991. Model' Doal'pijskogo zonal'nogo metamorfizma Bol'shogo Kavkaza [Model of the Pre-Alpine Zonal metamorphism in the Greater Caucasus]. In: Korikovsky, S.P (ed.) Petrologija metamorficheskih kompleksov Bol'shogo Kavkaza [Petrology of the Metamorphic Complexes of the Greater Caucasus]. Nauka, Moskva, 216-222. [In Russian].

Korikovsky, S.P., Shengelia, D., Chichinadze, G., Tsutsunava, T., Beridze, G., Javakhishvili, I. 2018. Contact Metamorphism of the Dizi Series (Greater Caucasus). In: $4^{\text {th }}$ International Scientific-Practical Conference on “Up-to-date Problems of Geology”, May 2018, Tbilisi, 55-57.

Kranidiotis, P., MacLean, W.H. 1987. Systematics of chlorite alteration at the Phelps Dodge massive sulfide deposit, Matagami, Quebec. Economic Geology 82, 1898-1911.

Kutelia, Z.A. 1983. Novye dannye po stratigrafii Dizskoj serii (Svanetija) [New data on the stratigraphy of the Dizi Series (Svaneti)]. Soobshhenija Akademii Nauk GSSR 109, 3, 29-33. [In Russian].

Lavrent'eva, I.V., Perchuk, L.L. 1981. Kordierit-granatovyj termometr [Cordierite-garnet thermometer]. Doklady Akademii Nauk SSSR 259, 3, 697-700. [In Russian].

Leven, E.J., Okay, A.I. 1996. Foraminifera from the exotic Permo-Carboniferous limestone blocks in the Karakaya Complex, Northwestern Turkey. Rivista Italiana di Paleontologia e Stratigrafia 102 (2), 139-174, https://doi.org/10.13130/2039-4942/5245

Meijers, M.J.M., Vrouwe, B., Van Hinsbergen, D.J.J., Kuiper, K.F., Wijbrans, J., Davies, G.R., Stephenson, R.A., Kaymakcı, N., Matenco, L., Saintot, A. 2010. Jurassic arc volcanism on Crimea (Ukraine): Implications for the paleo-subduction zone configuration of the Black Sea region. Lithos 119, 3-4, 412-426, https://doi.org/10.1016/j.lithos.2010.07.017

Novikov, E.A. 1963. Novye dannye o vozraste porod, vskrytyh glubokimi skvazhinami v stepnom Krymu [New data on the age of rocks exposed by deep wells in the steppe Crimea]. Vestnik LSU 18, Geologija $i$ Geografija 3, 12-23. [In Russian].

Okay, A.I., Topuz, G. 2016. Variscan orogeny in the Black Sea region. International Journal of Earth Sciences 106, 569-592, https://doi.org/10.1007/S00531-016-1395-Z

Okay, A.I., Noble, J., Tekin, U.K. 2011a. Devonian radiolarian ribbon cherts from the Karakaya Complex, northwest Turkey: implications for the PaleoTethyan evolution. Comptes Rendus Palevol 10, 1-10, https://doi.org/10.1016/j.crpv.2010.12.002

Okay, A.I., Altıner, D., Kılıç, A.M. 2015. Triassic lime- stone, turbidite and serpentinite Cimmeride orogeny in the Central Pontides. Geological Magazine 152, 3, 460-479, https://doi.org/10.1017/S0016756814000429

Perchuk, L.L., Ryabchikov, I.D. 1976. Fazovoe sootvetstvie $v$ mineral'nyh sistemah [Phase correspondence in mineral systems]. Nedra, Moskva, 287 pp. [In Russian].

Reverdatto, V.V. 1970. Facii kontaktovogo metamorfizma [The Facies of contact metamorphism]. Nedra, Moskva, 271 pp. [In Russian].

Savitsina, G.O., Plakhotny, L.G. 1965. Novye dannye o prirode Novocarinskoj gravitacionnoj anomalii [New data on the nature of the Novotsarinsk gravity anomaly]. Doklady Akademii Nauk SSSR 6, 108-111. [In Russian].

Shengelia, D., Tsutsunava, T., Chichinadze, G., Beridze, G. 2015. Akhali monatsemebi dizis seriis metamorp'izmis shesakheb [New Data on the Metamorphism of the Dizi Series]. Proceedings of Al. Janelidze Institute of Geology 127, 117-123. [In Georgian].

Shniukova, K.E. 2016. Magmatizm v zone sochlenenija Zapadnogo Prichernomor'ja, Gornogo Kryma i Skifskoj platformy [Magmatism in the junction zone of the Western Black sea, Mountainous Crimea and Scythian platform]. Naukova Dumka, Kyiv, 240 pp. [In Russian].

Shumlyanskyy, L.V., Shumlyanskyy, V.O. 2019. Preliminary data on $\mathrm{U}-\mathrm{Pb}$ isotope age of igneous rocks of Crimea, Ukraine. In: Conference dedicated to the $50^{\text {th }}$ Anniversary of the M.P. Semenenko Institute of Geochemistry, Mineralogy and Ore Formation, Kyiv, v. 1, 241-243.

Shumlyanskyy, L., Belousova, E., Petrenko, O. 2017. Geochemistry of zircons from basic rocks of the Korosten anorthosite-mangerite-charnockite-granite complex, North-Western region of the Ukrainian Shield. Mineralogy and Petrology 111, 459-466, https://doi.org/10.1007/s00710-017-0514-2

Shumlyanskyy, L.V., Stepanyuk, L.M., Claesson, S., Rudenko, K.V., Bekker, A.Yu. 2018. Uran-svynceva geohronologija granitoidiv za tsyrkonom ta monacytom Zhytomyrskogo ta Sheremetivskogo kompleksiv, Pivnichno-zahidnyj rajon Ukrainskogo shhyta [The $\mathrm{U}-\mathrm{Pb}$ zircon and monazite geochronology of granitoids of the Zhytomyr and Sheremetiv complexes, the Northwestern region of the Ukrainian Shield]. Mineralogical Journal (Ukraine) 40 (2), 63-85. [In Ukrainian], https://doi.org/10.15407/mineraljournal.40.02.063

Sláma, J., Košler, J., Condon, D.J., Crowley, J.L., Gerdes, A., Hanchar, J.M., Horstwood, M.S.A., Morris, G.A., Nasdala, L., Norberg, N., Schaltegger, U., Schoene, B., Tubrett, M.N. Whitehouse, M.J. 2008. Plešovice zircon - A new natural reference material for $\mathrm{U}-\mathrm{Pb}$ and $\mathrm{Hf}$ isotopic microanalysis. Chemical Geology 249, 1-35, https://doi.org/10.1016/j.chemgeo.2007.11.005

Slavin, V.I. 1960. Paleozojskie otlozhenija juzhnogo sklona Glavnogo Kavkazskogo hrebta [Paleozoic deposits of the southern slope of the Greater Caucasus Main Range]. Doklady Akademii Nauk SSSR 134, 5, 1184 1187. [In Russian]. 
Snegiryova, O.V., Shalya, A.A. 1966. Nekotorye osobennosti epigeneza i metamorfizma paleozojskih i mezozojskih otlozhenij Kryma [Some features of epigenesis and metamorphism of Paleozoic and Mesozoic rocks of the Crimea]. Izvestia Vuzov, Geologija i razvedka 10, 38-40. [In Russian].

Söderlund, U., Patchett, J.P., Vervoort, J.D., Isachsen, C.E. 2004. The ${ }^{176} \mathrm{Lu}$ decay constant determined by Lu-Hf and $\mathrm{U}-\mathrm{Pb}$ isotope systematics of Precambrian mafic intrusions. Earth and Planetary Science Letters 219, 311324, https://doi.org/10.1016/S0012-821X(04)00012-3

Somin, M. 1971. Dojurskoe osnovanie Glavnogo hrebta Juzhnogo sklona Bol'shogo Kavkaza [Pre-Jurassic basement of the Main Range and Southern Slope of the Greater Caucasus]. Nauka, Moskva, 245 pp. [In Russian].

Somin, M.L. 2011. Pre-Jurassic basement of the Greater Caucasus: Brief Overview. Turkish Journal of Earth Sciences 20, 545-610, https://doi.org/10.3906/yer-1008-6
Somin, M.L., Belov, A.A. 1967a. O stratigraficheskom raschlenenii Desskoj serii Svanetii (Central'nyj Kavkaz) [On the stratigraphic dismemberment of the Desi series of Svaneti (Central Caucasus)]. Bjulleten' Moskovskogo obshhestva ispytatelej prirody, Otdel geologicheskij 42, 1, 16-21. [In Russian].

Spear, F.S. 1981. Amphibole-plagioclase equilibria: an empirical model for the relation albite + tremolite $=$ edenite + quatz. Contributions to Mineralogy and Petrology 77, 4, 355-364, https://doi.org/10.1007/BF00371564

Stern, R.A., Bodorkos, S., Kamo, S.L., Hickman, A.H., Corfu, F. 2009. Measurement of SIMS instrumental mass fractionation of $\mathrm{Pb}$ isotopes during zircon dating. Geostandards and Geoanalytical Research 33, 145-168, https://doi.org/10.1111/j.1751-908X.2009.00023.x

Whitney, D., Evans, B. 2010. Abbreviations for names of rock-forming minerals. American Mineralogist 95, 185-187, https://doi.org/10.2138/am.2010.3371

Zang, W., Fyfe, W. 1995. Chloritization of the Hydrothermally Altered Bedrock at the Igarape-Bahia Gold Deposit, Carajas, Brazil. Mineralium Deposita 30, 30-38. 


\section{Supporting Online Material}

For the article

\section{METAMORPHISM OF THE ROCKS OF THE DIZI SERIES (THE GREATER CAUCASUS): PETROGRAPHY, MINERALOGY AND EVOLUTION OF METAMORPHIC ASSEMBLAGES}

Irakli Javakhishvili, David Shengelia, Leonid Shumlyanskyy, Tamara Tsutsunava, Giorgi Chichinadze, Giorgi Beridze

PDF file includes:

Supplement 1: Folded hornfelses. Enguri river valley, Zugdidi-Mestia highway.

Supplement 2: Results of electron microprobe analyses of minerals from rocks of the Dizi Series.

Supplement 3: Diorite-porphyrite of the Dizi Series (the Khumpreri river gorge)

Supplement 4: Contact of the intrusion with the regionally metamorphosed rocks of the Dizi Series.

Supplement 5: Equilibrium mineral parageneses of the III zone of contact metamorphism.

Supplement 6: Idiomorphic poikiloblasts of green hornblende in xenoliths. 


\section{Supporting Online Material}

Supplement 1. Folded hornfelses. Enguri river valley, Zugdidi-Mestia highway.

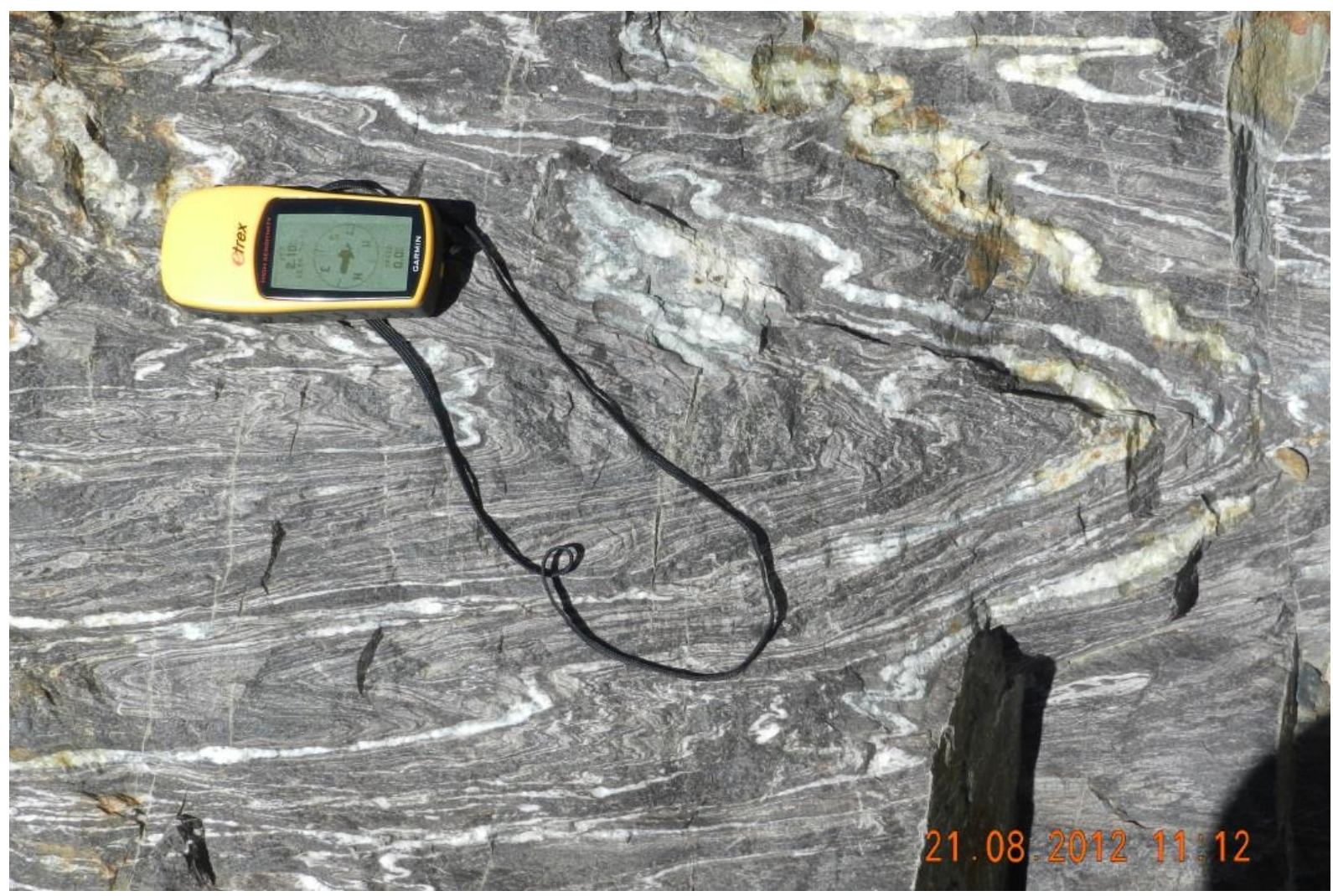


Supplement 2. Results of electron microprobe analyses of minerals (wt. \%) from rocks of the Dizi Series.

\begin{tabular}{|c|c|c|c|c|c|c|c|c|c|c|c|c|c|c|}
\hline & \multicolumn{9}{|c|}{ Muscovite } & \multicolumn{5}{|c|}{ Biotite } \\
\hline & $4-13$ & $8-13$ & $15-13$ & $15-13$ & 50-12 & $119-12$ & $119-12$ & 94-12 & 94-12 & $15-13$ & $\begin{array}{c}15- \\
13 / 1\end{array}$ & $50-12$ & $50-12$ & $75-13 / 1$ \\
\hline $\mathrm{SiO} 2$ & 47.42 & 49.81 & 46.58 & 48.39 & 47.21 & 47.2 & 47.38 & 46.03 & 46.25 & 36.86 & 35.58 & 37 & 34.55 & 35.51 \\
\hline TiO2 & 0.28 & 0.02 & 0.76 & 0.19 & 0.68 & 0.71 & 0.44 & 0.58 & 0.9 & 1.62 & 1.87 & 1.61 & 2.28 & 3.04 \\
\hline Al2O3 & 32.87 & 36.6 & 35.89 & 32.23 & 35.53 & 34.22 & 33 & 36.02 & 35.85 & 17.42 & 17.76 & 17.93 & 21.67 & 16.11 \\
\hline $\mathrm{FeO}$ & 2.01 & 1.3 & 1.35 & 1.75 & 1.09 & 1.01 & 1.28 & 0.9 & 1.02 & 18.01 & 18.84 & 16.68 & 16.98 & 17.99 \\
\hline MnO & - & 0.04 & 0.02 & 0 & - & - & 0.02 & 0.01 & - & 0.42 & 0.27 & 0.22 & 0.22 & 0.11 \\
\hline MgO & 1.99 & 1 & 0.89 & 1.99 & 1.09 & 0.96 & 1.28 & 0.38 & 0.44 & 12.09 & 11.28 & 12.82 & 9.86 & 10.25 \\
\hline $\mathrm{CaO}$ & 0.01 & 0.06 & 0.01 & 0.06 & 0.03 & 0.05 & 0.08 & - & 0.02 & 0.09 & 0.1 & 0.02 & 0.02 & 0.1 \\
\hline $\mathrm{Na} 2 \mathrm{O}$ & 0.28 & 0.02 & 0.77 & 0.21 & 1.04 & 0.4 & 0.34 & 0.51 & 0.48 & 0.07 & 0.14 & 0.14 & 0.21 & 0.05 \\
\hline K2O & 10.06 & 9.51 & 9.69 & 9.64 & 9.4 & 9.68 & 9.56 & 9.74 & 10.16 & 8.27 & 8.92 & 8.88 & 9.64 & 9.68 \\
\hline $\mathbf{F}$ & - & - & - & - & - & - & - & - & - & 0.77 & 0.73 & 0.68 & 0.29 & 0.61 \\
\hline Cl & - & - & - & - & - & - & - & - & - & 0.07 & 0.08 & 0.04 & 0.02 & 0.12 \\
\hline Total & 94.92 & 98.36 & 95.96 & 94.46 & 96.07 & 94.23 & 93.38 & 94.17 & 95.12 & 95.69 & 95.57 & 96.02 & 95.74 & 93.57 \\
\hline $\mathbf{S i}$ & 3.18 & 3.23 & 3.08 & 3.27 & 3.11 & 3.19 & 3.23 & 3.11 & 3.1 & 2.87 & 2.78 & 2.84 & 2.67 & 2.85 \\
\hline $\mathbf{T i}$ & 0.01 & - & 0.04 & 0.01 & 0.03 & 0.04 & 0.02 & 0.03 & 0.05 & 0.09 & 0.11 & 0.09 & 0.13 & 0.18 \\
\hline Al & 2.6 & 2.8 & 2.8 & 2.56 & 2.76 & 2.73 & 2.65 & 2.87 & 2.83 & 1.6 & 1.63 & 1.62 & 1.97 & 1.52 \\
\hline $\mathrm{Al}, 4$ & - & - & - & - & - & - & - & - & - & 1.13 & 1.22 & 1.16 & 1.33 & 1.15 \\
\hline Al, 6 & - & - & - & - & - & - & - & - & - & 0.47 & 0.41 & 0.46 & 0.64 & 0.37 \\
\hline $\mathrm{Fe}^{\prime \prime}$ & 0.11 & 0.07 & 0.07 & 0.1 & 0.06 & 0.06 & 0.07 & 0.05 & 0.06 & 1.17 & 1.23 & 1.07 & 1.09 & 1.21 \\
\hline Mn & - & - & - & - & - & - & - & - & - & 0.03 & 0.02 & 0.01 & 0.01 & 0.01 \\
\hline $\mathbf{M g}$ & 0.2 & 0.1 & 0.09 & 0.2 & 0.11 & 0.1 & 0.13 & 0.04 & 0.04 & 1.4 & 1.31 & 1.47 & 1.13 & 1.23 \\
\hline $\mathrm{Ca}$ & - & - & 0 & 0 & - & - & 0.01 & - & - & 0.01 & 0.01 & - & - & 0.01 \\
\hline $\mathrm{Na}$ & 0.04 & - & 0.01 & 0.03 & 0.13 & 0.05 & 0.04 & 0.07 & 0.06 & 0.01 & 0.02 & 0.02 & 0.03 & 0.01 \\
\hline $\mathbf{K}$ & 0.86 & 0.79 & 0.82 & 0.83 & 0.79 & 0.83 & 0.83 & 0.84 & 0.87 & 0.82 & 0.89 & 0.87 & 0.95 & 0.99 \\
\hline $\mathbf{F}$ & - & - & - & - & - & - & - & - & - & 0.19 & 0.18 & 0.17 & 0.07 & 0.15 \\
\hline $\mathrm{Cl}$ & - & - & - & - & - & - & - & - & - & 0.01 & 0.01 & 0.01 & - & 0.02 \\
\hline $\mathbf{O H}$ & - & - & - & - & - & - & - & - & - & 1.8 & 1.81 & 1.83 & 1.93 & 1.83 \\
\hline $\mathbf{X}(\mathbf{M g})$ & - & - & - & - & - & - & - & - & - & 0.54 & 0.51 & 0.58 & 0.51 & 0.5 \\
\hline
\end{tabular}




\begin{tabular}{|c|c|c|c|c|c|c|c|c|c|c|c|c|c|c|c|}
\hline & \multicolumn{8}{|c|}{ Biotite } & \multicolumn{5}{|c|}{ Mg-Hornblende } & \multicolumn{2}{|c|}{ Actinolite } \\
\hline & 119-12 & $94-12$ & $91-12$ & $91-12$ & $11-15 / 2$ & $11-15 / 3$ & $62-12$ & $62-12$ & $15-13 / 1$ & $15-13 / 1$ & $50-12 / 1$ & $11-15 / 3$ & $62-12$ & $41-12$ & $41-12$ \\
\hline $\mathrm{SiO} 2$ & 34.12 & 34.29 & 35.45 & 34.18 & 36.79 & 38.15 & 36.54 & 36.45 & 47.93 & 45.37 & 46.5 & 47.81 & 46.4 & 50.95 & 49.85 \\
\hline TiO2 & 2.83 & 1.37 & 1.41 & 1.62 & 3.17 & 1.87 & 4.22 & 4.41 & 0.4 & 0.47 & 0.4 & 0.3 & 1.25 & 0.22 & 0.26 \\
\hline $\mathrm{Al2O3}$ & 20.98 & 21.91 & 21.8 & 21.68 & 14.53 & 16.39 & 14.34 & 14.21 & 8.02 & 11.4 & 9.75 & 8.15 & 7.61 & 3.91 & 5.86 \\
\hline $\mathrm{FeO}$ & 18.73 & 21.23 & 21.45 & 19.93 & 15.77 & 15.23 & 19.58 & 19.17 & 16.59 & 15.36 & 14.3 & 13.2 & 16.41 & 14.79 & 15.29 \\
\hline MnO & 0.14 & 0.06 & 0.12 & 0.04 & 0.17 & 0.07 & 0.22 & 0.2 & 0.95 & 0.07 & 0.19 & 0.31 & 0.41 & 0.54 & 0.63 \\
\hline MgO & 9.12 & 6.77 & 6.78 & 7.42 & 16.84 & 15.55 & 10.88 & 10.95 & 11.01 & 10.31 & 12.28 & 14.12 & 11.5 & 12.19 & 12.61 \\
\hline $\mathrm{CaO}$ & - & - & 0.01 & 0.1 & 0.08 & 0.05 & 0.03 & 0.04 & 12.37 & 12.17 & 11.75 & 11.41 & 11.2 & 12.38 & 12.4 \\
\hline $\mathrm{Na} 2 \mathrm{O}$ & 0.22 & 0.17 & 0.17 & 0.19 & 0.17 & 0.26 & 0.04 & 0.07 & 0.49 & 0.79 & 0.86 & 0.98 & 1.41 & 0.23 & 0.32 \\
\hline K2O & 9.34 & 9.31 & 9.26 & 9.08 & 9.2 & 8.11 & 9.73 & 9.59 & 0.45 & 0.64 & 0.6 & 0.26 & 0.74 & 0.19 & 0.29 \\
\hline $\mathbf{F}$ & 0.43 & 0.49 & 0.56 & 0.52 & 0.13 & 0.53 & 0.62 & 0.64 & - & - & - & - & - & - & - \\
\hline Cl & 0.02 & - & 0.06 & 0.05 & - & 0.07 & 0.05 & 0.04 & - & - & - & - & - & - & - \\
\hline Total & 95.93 & 95.6 & 97.07 & 94.81 & 96.85 & 96.28 & 96.25 & 95.77 & 98.21 & 96.58 & 96.63 & 96.54 & 85.73 & 95.4 & 97.51 \\
\hline $\mathbf{S i}$ & 2.66 & 2.71 & 2.77 & 2.71 & 2.75 & 2.89 & 2.87 & 2.88 & 7.07 & 6.76 & 6.99 & 6.99 & 7.52 & 7.62 & 7.32 \\
\hline $\mathbf{T i}$ & 0.17 & 0.08 & 0.08 & 0.1 & 0.18 & 0.11 & 0.25 & 0.26 & 0.04 & 0.05 & 0.04 & 0.03 & 0.15 & 0.02 & 0.03 \\
\hline Al & 1.93 & 2.04 & 2.01 & 2.03 & 1.28 & 1.46 & 1.33 & 1.32 & - & - & - & - & - & - & - \\
\hline $\mathrm{Al}, 4$ & 1.34 & 1.29 & 1.23 & 1.29 & 1.25 & 1.11 & 1.13 & 1.12 & 0.93 & 1.24 & 1.01 & 1.01 & 0.48 & 0.38 & 0.68 \\
\hline Al, 6 & 0.59 & 0.75 & 0.77 & 0.74 & 0.04 & 0.35 & 0.2 & 0.2 & 0.46 & 0.76 & 1.39 & 0.39 & 0.98 & 0.31 & 0.33 \\
\hline $\mathrm{Fe}^{\prime \prime \prime}$ & - & - & - & - & - & - & - & - & 0.19 & 0.1 & 0.44 & 0.44 & 0.46 & 0.07 & 0.18 \\
\hline $\mathrm{Fe}^{\prime \prime}$ & 1.22 & 1.4 & 1.4 & 1.32 & 0.99 & 0.96 & 1.28 & 1.26 & 1.85 & 1.82 & 1.04 & 1.17 & 1.77 & 1.78 & 1.7 \\
\hline Mn & 0.01 & - & 0.01 & - & 0.01 & - & 0.01 & 0.01 & 0.12 & - & 0.02 & 0.04 & 0.06 & 0.07 & 0.08 \\
\hline $\mathbf{M g}$ & 1.06 & 0.8 & 0.79 & 0.88 & 1.88 & 1.75 & 1.27 & 1.29 & 2.42 & 2.29 & 3.07 & 3.07 & 2.78 & 2.72 & 2.76 \\
\hline $\mathrm{Ca}$ & - & - & - & 0.01 & 0.01 & - & - & - & 1.95 & 1.94 & 1.86 & 1.79 & 1.74 & 1.98 & 1.95 \\
\hline $\mathrm{Na}$ & 0.03 & 0.03 & 0.03 & 0.03 & 0.02 & 0.04 & 0.01 & 0.01 & 0.14 & 0.22 & 0.25 & 0.28 & 0.44 & 0.07 & 0.09 \\
\hline $\mathbf{K}$ & 0.93 & 0.94 & 0.92 & 0.92 & 0.88 & 0.78 & 0.97 & 0.96 & 0.08 & 0.12 & 0.12 & 0.05 & 0.15 & 0.04 & 0.05 \\
\hline $\mathbf{F}$ & 0.11 & 0.12 & 0.14 & 0.13 & 0.03 & 0.13 & 0.15 & 0.16 & - & - & - & - & - & - & - \\
\hline $\mathrm{Cl}$ & - & - & 0.01 & 0.01 & - & 0.01 & 0.01 & 0.01 & - & - & - & - & - & - & - \\
\hline $\mathbf{O H}$ & 1.89 & 1.88 & 1.85 & 1.86 & 1.97 & 1.86 & 1.84 & 1.84 & - & - & - & - & - & - & - \\
\hline $\mathbf{X}(\mathbf{M g})$ & 0.46 & 0.36 & 0.36 & 0.4 & 0.65 & 0.64 & 0.5 & 0.5 & 0.54 & 0.54 & 0.5 & 0.66 & 0.56 & 0.59 & 0.68 \\
\hline
\end{tabular}




\begin{tabular}{|c|c|c|c|c|c|c|c|c|c|c|c|c|c|c|c|}
\hline & \multicolumn{7}{|c|}{ Actinolite } & \multicolumn{4}{|c|}{ Clinopyroxene } & \multicolumn{4}{|c|}{ Cummingtonite } \\
\hline & $45-12$ & $75-13 / 1$ & $75-13 / 1$ & $11-15 / 2$ & $11-15 / 2$ & $11-15 / 2$ & $11-15 / 2$ & $41-12$ & $75-13-1$ & $62-12$ & $62-12$ & $11-15 / 2$ & $11-15 / 2$ & $11-15 / 3$ & $11-15 / 3$ \\
\hline $\mathrm{SiO} 2$ & 50.9 & 50.91 & 51.99 & 50.83 & 49.54 & 50.99 & 49.62 & 52.07 & 50.44 & 51.34 & 51.55 & 54.19 & 54.36 & 54.22 & 53.9 \\
\hline TiO2 & 0.09 & 0.07 & 0.1 & 0.37 & 0.51 & 0.24 & 0.42 & 0.16 & 0.05 & 0.06 & 0.05 & 0.08 & 0.09 & 0.07 & 0.1 \\
\hline $\mathrm{Al2O3}$ & 3.12 & 3.02 & 3.3 & 3.86 & 5.58 & 4.39 & 5.81 & 0.98 & 0.64 & 0.64 & 0.37 & 1.05 & 1.04 & 0.69 & 1.38 \\
\hline $\mathrm{FeO}$ & 16.45 & 19.17 & 14.4 & 12.73 & 13.42 & 12.87 & 12.8 & 13.3 & 15.12 & 14.45 & 14.34 & 20.75 & 20.92 & 20.82 & 20.74 \\
\hline MnO & 0.21 & 0.45 & 0.19 & 0.56 & 0.59 & 0.36 & 0.36 & 0.87 & 0.45 & 0.72 & 0.91 & 0.91 & 0.9 & 0.78 & 0.73 \\
\hline MgO & 11.88 & 10.59 & 13.78 & 18.17 & 17.39 & 18 & 17.57 & 9.79 & 8.12 & 9.07 & 8.34 & 21.21 & 21.28 & 19.03 & 18.62 \\
\hline $\mathrm{CaO}$ & 12.4 & 12.23 & 12.64 & 10.97 & 10.63 & 11.47 & 11.66 & 23.9 & 24.17 & 23.37 & 23.96 & 1.41 & 1.59 & 1.09 & 1.62 \\
\hline $\mathrm{Na} 2 \mathrm{O}$ & 0.33 & 0.3 & 0.36 & 0.31 & 0.48 & 0.37 & 0.41 & 0.06 & 0.15 & 0.22 & 0.11 & 0.11 & 0.12 & 0.07 & 0.16 \\
\hline K2O & 0.21 & 0.23 & 0.18 & 0.19 & 0.29 & 0.17 & 0.23 & - & - & 0.01 & - & - & - & - & 0.02 \\
\hline Total & 95.59 & 96.97 & 96.94 & 97.99 & 98.43 & 98.86 & 98.88 & 100.17 & 99.16 & 99.92 & 99.63 & 99.71 & 100.3 & 96.77 & 97.27 \\
\hline $\mathbf{S i}$ & 7.48 & 7.65 & 7.63 & 7.39 & 7.16 & 7.34 & 7.14 & 1.97 & 1.97 & 1.98 & 2 & 7.6 & 7.58 & 7.91 & 7.82 \\
\hline Ti & 0.02 & 0.01 & 0.01 & 0.04 & 0.06 & 0.03 & 0.05 & - & - & - & - & 0.01 & 0.01 & 0.01 & 0.01 \\
\hline Al & - & - & - & - & - & - & - & 0.04 & 0.03 & 0.03 & 0.02 & 0.17 & 0.17 & 0.12 & 0.24 \\
\hline $\mathrm{Al}, 4$ & 0.52 & 0.35 & 0.37 & 0.61 & 0.84 & 0.66 & 0.86 & - & - & - & - & - & - & - & - \\
\hline Al, 6 & 0.26 & 0.18 & 0.2 & 0.06 & 0.11 & 0.08 & 0.12 & 0.02 & - & 0.01 & - & - & - & - & - \\
\hline $\mathrm{Fe}^{\prime \prime \prime}$ & 0.09 & 0.06 & 0.03 & - & 0.28 & 0.11 & 0.28 & - & 0.04 & 0.03 & - & 0.63 & 0.67 & 0.06 & 0.14 \\
\hline $\mathrm{Fe}^{\prime \prime}$ & 1.72 & 2.34 & 1.74 & 1.55 & 1.34 & 1.43 & 1.25 & 0.42 & 0.45 & 0.44 & 0.46 & 1.8 & 1.76 & 2.48 & 2.37 \\
\hline Mn & - & 0.06 & 0.02 & 0.07 & 0.07 & 0.04 & 0.04 & 0.03 & 0.01 & 0.02 & 0.03 & 0.11 & 0.11 & 0.1 & 0.09 \\
\hline Mg & 2.89 & 2.37 & 3.01 & 3.94 & 3.74 & 3.86 & 3.76 & 0.55 & 0.47 & 0.52 & 0.48 & 4.43 & 4.42 & 4.14 & 4.03 \\
\hline $\mathrm{Ca}$ & 1.32 & 1.97 & 1.99 & 1.71 & 1.65 & 1.77 & 1.8 & 0.97 & 1.01 & 0.96 & 1 & 0.21 & 0.24 & 0.17 & 0.25 \\
\hline $\mathrm{Na}$ & 0.1 & 0.09 & 0.1 & 0.09 & 0.13 & 0.1 & 0.11 & - & 0.01 & 0.02 & 0.01 & 0.03 & 0.03 & 0.02 & 0.04 \\
\hline $\mathbf{K}$ & 0.04 & 0.04 & 0.03 & 0.04 & 0.05 & 0.03 & 0.04 & - & - & - & - & - & - & - & - \\
\hline $\mathbf{X}(\mathbf{M g})$ & 0.52 & 0.5 & 0.63 & 0.7 & 0.71 & 0.71 & 0.71 & 0.55 & 0.5 & 0.53 & 0.49 & 0.65 & 0.65 & 0.62 & 0.62 \\
\hline
\end{tabular}




\begin{tabular}{|c|c|c|c|c|c|c|c|c|c|c|c|c|c|c|c|c|}
\hline & \multicolumn{9}{|c|}{ Cordierite } & \multicolumn{4}{|c|}{ Chlorite } & \multicolumn{2}{|c|}{ Garnet } & \multirow{2}{*}{$\begin{array}{c}\text { Clinozoisite } \\
62-12\end{array}$} \\
\hline & $50-12$ & $119-12$ & $119-12$ & $119-12$ & $94-12$ & $94-12$ & 91-12 & $91-12$ & $91-12$ & $4-13$ & $8-13$ & $23-13$ & $50-12$ & $62-12$ & $62-12$ & \\
\hline $\mathrm{SiO} 2$ & 48.84 & 48.48 & 49.22 & 48.81 & 47.71 & 47.69 & 47.86 & 47.76 & 48.02 & 25.7 & 26.69 & 27.08 & 29.58 & 36.78 & 36.92 & 38.58 \\
\hline TiO2 & 0.36 & 0.01 & 0.04 & 0.04 & 0.05 & 0.01 & 0.04 & 0.04 & 0.03 & 0.06 & 0.06 & 0.9 & 0.11 & 2.11 & 2 & 0.05 \\
\hline $\mathrm{Al} 203$ & 32.72 & 32.47 & 33.38 & 32.97 & 32.47 & 32.22 & 32.4 & 32.14 & 32.6 & 22.57 & 23.34 & 21.11 & 19.44 & 13.78 & 13.49 & 28.86 \\
\hline $\mathrm{FeO}$ & 7.32 & 7.68 & 7.83 & 7.63 & 11.22 & 11.13 & 10.6 & 10.76 & 11.43 & 26.11 & 23.55 & 23.1 & 21.48 & 10.42 & 10.7 & 6.58 \\
\hline MnO & 0.03 & 0.25 & 0.26 & 0.2 & 0.14 & 0.23 & 0.26 & 0.23 & 0.27 & 0.21 & 0.11 & 0.24 & 0.39 & 0.67 & 0.53 & 0.27 \\
\hline $\mathrm{MgO}$ & 6.22 & 8.19 & 8.23 & 7.95 & 5.94 & 5.88 & 6.37 & 6.24 & 6.09 & 15.78 & 16.22 & 16.32 & 16.47 & 0.09 & 0.09 & 0.03 \\
\hline $\mathrm{CaO}$ & 0.04 & 0.04 & 0.04 & 0.05 & 0.03 & 0.05 & 0.03 & 0.03 & 0.02 & 0.02 & 0.02 & 0.07 & 0.12 & 34.15 & 34.27 & 24.06 \\
\hline $\mathrm{Na2O}$ & 2.3 & 0.2 & 0.15 & 0.14 & 0.17 & 0.25 & 0.19 & 0.17 & 0.19 & - & 0.01 & 0.01 & 0.02 & - & - & - \\
\hline K2O & 0.02 & 0.01 & 0.02 & 0.4 & 0 & 0.01 & 0.03 & 0.01 & 0.03 & 0.09 & 0 & 0.05 & 0.1 & - & - & - \\
\hline Total & 97.85 & 97.33 & 99.17 & 98.19 & 97.73 & 97.47 & 97.78 & 97.38 & 98.68 & 90.54 & 90.06 & 88.88 & 87.75 & 98 & 98 & 98.43 \\
\hline $\mathbf{S i}$ & 4.99 & 5.03 & 5.02 & 5.02 & 5.01 & 5.02 & 5.01 & 5.03 & 5 & 5.25 & 5.43 & 5.76 & 6.18 & 2.93 & 2.94 & 2.96 \\
\hline $\mathbf{T i}$ & 0.03 & - & - & - & - & - & - & - & - & 0.01 & 0.01 & 0.02 & 0.02 & 0.13 & 0.12 & - \\
\hline Al & 3.94 & 3.97 & 4.01 & 4 & 4.02 & 4 & 4 & 3.99 & 4 & 5.43 & 5.6 & 5.14 & 4.79 & 1.29 & 1.26 & 2.61 \\
\hline $\mathrm{Al}, 4$ & 1.01 & 0.97 & 0.98 & 0.98 & 0.99 & 0.98 & 0.99 & 0.97 & 1 & - & - & - & - & - & - & - \\
\hline Al, 6 & 2.94 & 3 & 3.02 & 3.02 & 3.03 & 3.02 & 3.01 & 3.01 & 2.99 & - & - & - & - & - & - & - \\
\hline $\mathrm{Cr}$ & - & - & - & - & - & - & - & - & - & - & 0.01 & - & 0.01 & - & - & - \\
\hline $\mathrm{Fe}^{\prime \prime \prime}$ & - & - & - & - & - & - & - & - & - & 0.08 & - & 0.03 & - & 0.61 & 0.62 & 0.46 \\
\hline $\mathrm{Fe}^{\prime \prime}$ & 0.62 & 0.67 & 0.67 & 0.66 & 0.98 & 0.98 & 0.93 & 0.95 & 0.99 & 4.37 & 4 & 3.96 & 3.75 & 0.09 & 0.09 & - \\
\hline Mn & - & 0.02 & 0.02 & 0.02 & 0.01 & 0.02 & 0.02 & 0.02 & 0.02 & 0.04 & 0.02 & 0.04 & 0.07 & 0.05 & 0.04 & 0.02 \\
\hline Mg & 0.95 & 1.27 & 1.25 & 1.22 & 0.93 & 0.92 & 0.99 & 0.98 & 0.94 & 4.8 & 4.92 & 4.86 & 5.13 & 0.01 & 0.01 & - \\
\hline $\mathrm{Ca}$ & - & - & - & 0.01 & - & 0.01 & - & - & - & - & - & 0.02 & 0.03 & 2.91 & 2.92 & 1.98 \\
\hline $\mathbf{N a}$ & 0.46 & 0.04 & 0.03 & 0.03 & 0.03 & 0.05 & 0.04 & 0.03 & 0.04 & - & - & - & 0.01 & - & - & - \\
\hline $\mathbf{K}$ & - & - & - & 0.05 & - & - & - & - & - & 0.02 & - & 0.01 & 0.03 & - & - & - \\
\hline $\mathbf{X}(\mathbf{M g})$ & 0.6 & 0.65 & 0.64 & 0.64 & 0.48 & 0.48 & 0.51 & 0.5 & 0.48 & 0.48 & 0.45 & 0.56 & 0.42 & 0.01 & 0.01 & - \\
\hline
\end{tabular}




\begin{tabular}{|c|c|c|c|c|c|c|c|c|c|c|c|c|c|c|c|c|}
\hline & \multicolumn{2}{|c|}{ Albite } & \multicolumn{7}{|c|}{ Andesine } & \multicolumn{2}{|c|}{ Labradorite } & \multicolumn{2}{|c|}{ Bytownite } & \multirow{2}{*}{$\begin{array}{c}\text { K-Feldspar } \\
94-12\end{array}$} & \multicolumn{2}{|c|}{ Prehnite } \\
\hline & $4-13$ & $4-13$ & $15-13$ & $50-12$ & $50-12$ & 75-13-1 & $11-15 / 2$ & $62-12$ & $62-12$ & 75-13-1 & $62-12$ & $75-13-1$ & $15-13-1$ & & $41-12$ & 41-12 \\
\hline $\mathrm{SiO} 2$ & 68.0 & 68.54 & 55.9 & 56.68 & 58.16 & 57.95 & 55.25 & 55.58 & 58.78 & 53.87 & 53.94 & 48.55 & 47.48 & 64.76 & 43.64 & 43.38 \\
\hline TiO2 & - & - & - & - & - & - & - & - & - & - & - & - & - & - & 0.02 & 0.01 \\
\hline $\mathrm{Al2O3}$ & 19.77 & 21.29 & 27.86 & 26.26 & 26.05 & 26.37 & 28.35 & 27.99 & 25.95 & 28.92 & 29.16 & 32.67 & 34.32 & 18.81 & 24.32 & 24.54 \\
\hline $\mathrm{FeO}$ & 0.1 & 0.04 & 0.26 & 0.23 & 0.29 & 0.11 & 0.35 & 0.33 & 0.15 & 0.04 & 0.46 & 0.21 & 0.13 & 0.27 & 0.41 & 0.57 \\
\hline MnO & - & - & - & - & - & - & - & - & - & - & - & - & - & - & 0.04 & 0.19 \\
\hline MgO & - & - & - & - & - & - & - & - & - & - & - & - & - & - & 0.01 & - \\
\hline $\mathrm{CaO}$ & 0.04 & 1.36 & 9.75 & 7.54 & 7.34 & 8.52 & 10.34 & 9.85 & 7.62 & 11.65 & 11.32 & 16.08 & 16.65 & 0.6 & 27.34 & 27.3 \\
\hline $\mathrm{Na2O}$ & 11.41 & 9.53 & 6.37 & 7.52 & 7.59 & 6.61 & 6.02 & 6.02 & 7.28 & 5.07 & 5.23 & 2.46 & 2 & 0.2 & - & - \\
\hline K2O & 0.07 & 0.01 & 0.08 & 0.16 & 0.19 & 0.2 & 0.03 & 0.12 & 0.15 & 0.12 & 0.13 & 0.21 & 0.07 & 16.06 & - & - \\
\hline Total & 99.39 & 100.77 & 100.22 & 98.39 & 99.62 & 99.76 & 100.34 & 99.89 & 99.93 & 99.67 & 100.24 & 100.18 & 100.65 & 100.7 & 95.78 & 95.99 \\
\hline $\mathbf{S i}$ & 2.99 & 3.02 & 2.5 & 2.56 & 2.6 & 2.6 & 2.47 & 2.5 & 2.63 & 2.44 & 2.43 & 2.22 & 2.16 & 2.98 & 2.73 & 2.71 \\
\hline $\mathbf{T i}$ & - & - & - & - & - & - & - & - & - & - & - & - & - & - & - & - \\
\hline Al & 1.03 & 1.1 & 1.47 & 1.4 & 1.37 & 1.4 & 1.5 & 1.48 & 1.37 & 1.54 & 1.55 & 1.76 & 1.84 & 1.02 & 1.79 & 1.81 \\
\hline $\mathrm{Fe}^{\prime \prime}$ & - & - & 0.01 & 0.01 & 0.01 & - & 0.01 & 0.01 & 0.01 & - & 0.02 & 0.01 & - & 0.01 & 0.02 & 0.03 \\
\hline Mn & - & - & - & - & - & - & - & - & - & - & - & - & - & - & - & 0.01 \\
\hline Mg & - & - & - & - & - & - & - & - & - & - & - & - & - & - & - & - \\
\hline Ca & - & 0.06 & 0.47 & 0.36 & 0.35 & 0.41 & 0.5 & 0.47 & 0.36 & 0.56 & 0.55 & 0.79 & 0.81 & 0.03 & 1.83 & 1.83 \\
\hline $\mathrm{Na}$ & 0.97 & 0.81 & 0.55 & 0.66 & 0.66 & 0.58 & 0.52 & 0.52 & 0.63 & 0.44 & 0.46 & 0.22 & 0.18 & 0.02 & - & - \\
\hline $\mathbf{K}$ & - & - & - & 0.01 & 0.01 & 0.01 & - & 0.01 & 0.01 & 0.01 & 0.01 & 0.01 & - & 0.94 & - & - \\
\hline $\mathbf{X}(\mathbf{C a})$ & - & 0.07 & 0.46 & 0.35 & 0.34 & 0.41 & 0.49 & 0.47 & 0.36 & 0.56 & 0.54 & 0.77 & 0.82 & 0.03 & - & - \\
\hline $\mathbf{X}(\mathbf{N a})$ & 0.99 & 0.93 & 0.54 & 0.64 & 0.64 & 0.58 & 0.51 & 0.52 & 0.63 & 0.44 & 0.45 & 0.21 & 0.18 & 0.02 & - & - \\
\hline $\mathbf{X}(\mathbf{K})$ & - & - & - & 0.01 & 0.01 & 0.01 & - & 0.01 & 0.01 & 0.01 & 0.01 & 0.01 & - & 0.95 & - & - \\
\hline
\end{tabular}


Supplement 3. Diorite-porphyrite of the Dizi Series (the Khumpreri river gorge): XPL.

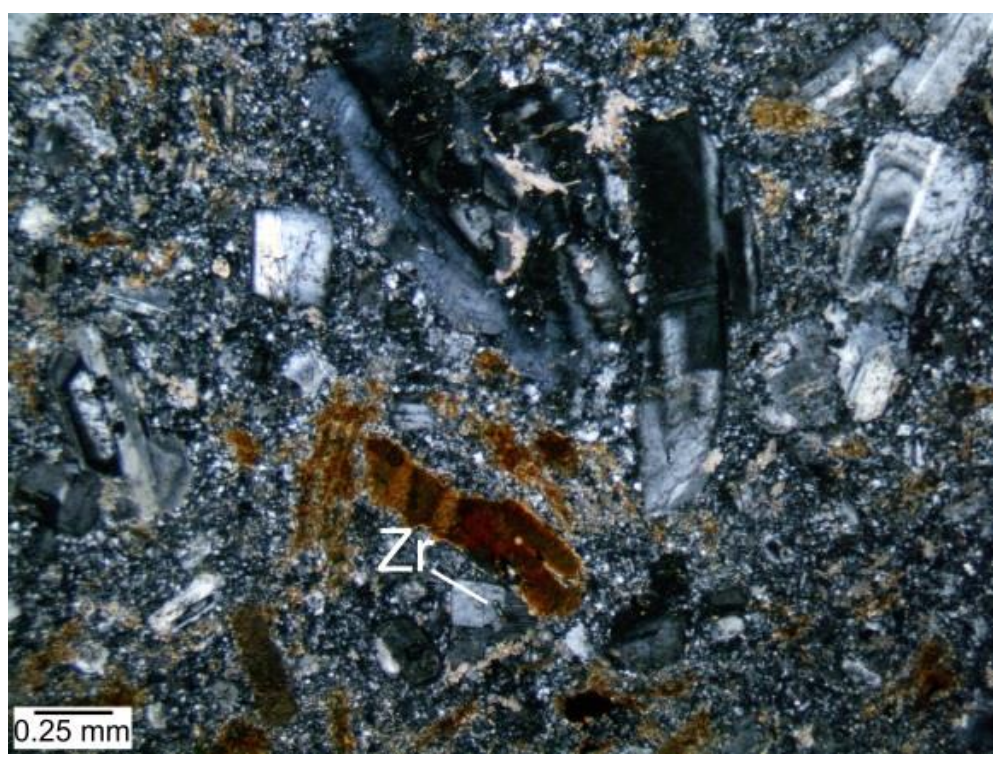

Supplement 4. Contact of the intrusion with the deformed and regionally metamorphosed rocks of the Dizi Series.

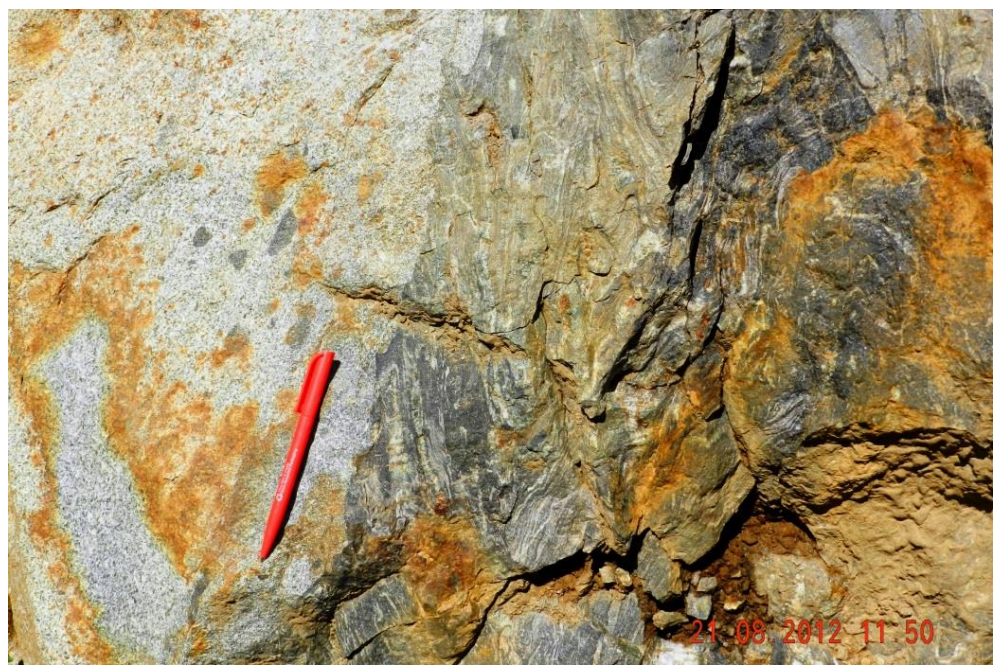


Supplement 5. Equilibrium mineral parageneses in the hornfels of the III zone of contact metamorphism: a, c PPL and $b, d-$ XPL.

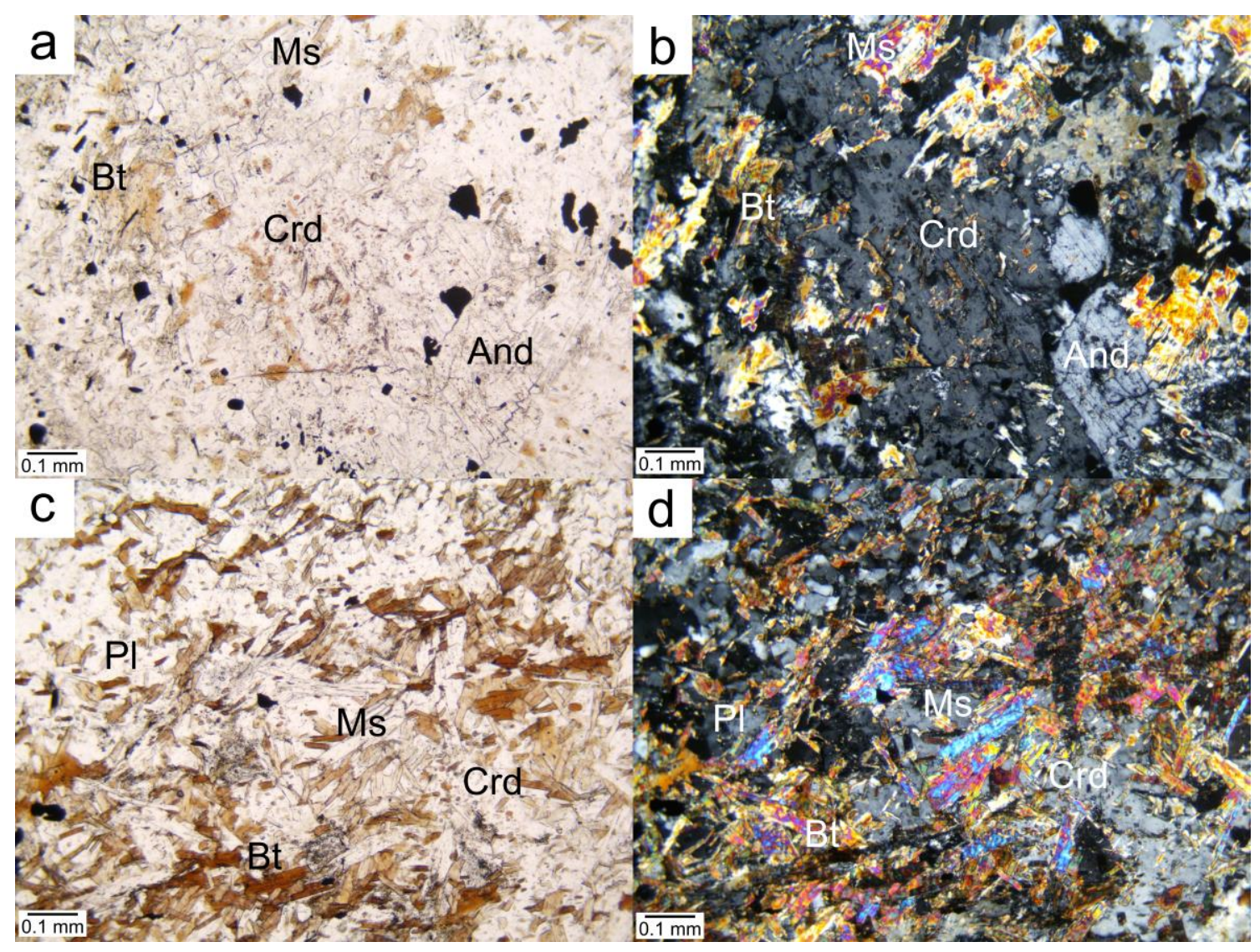

Supplement 6. Idiomorphic poikiloblasts of green hornblende in xenoliths: a - PPL and b - XPL.

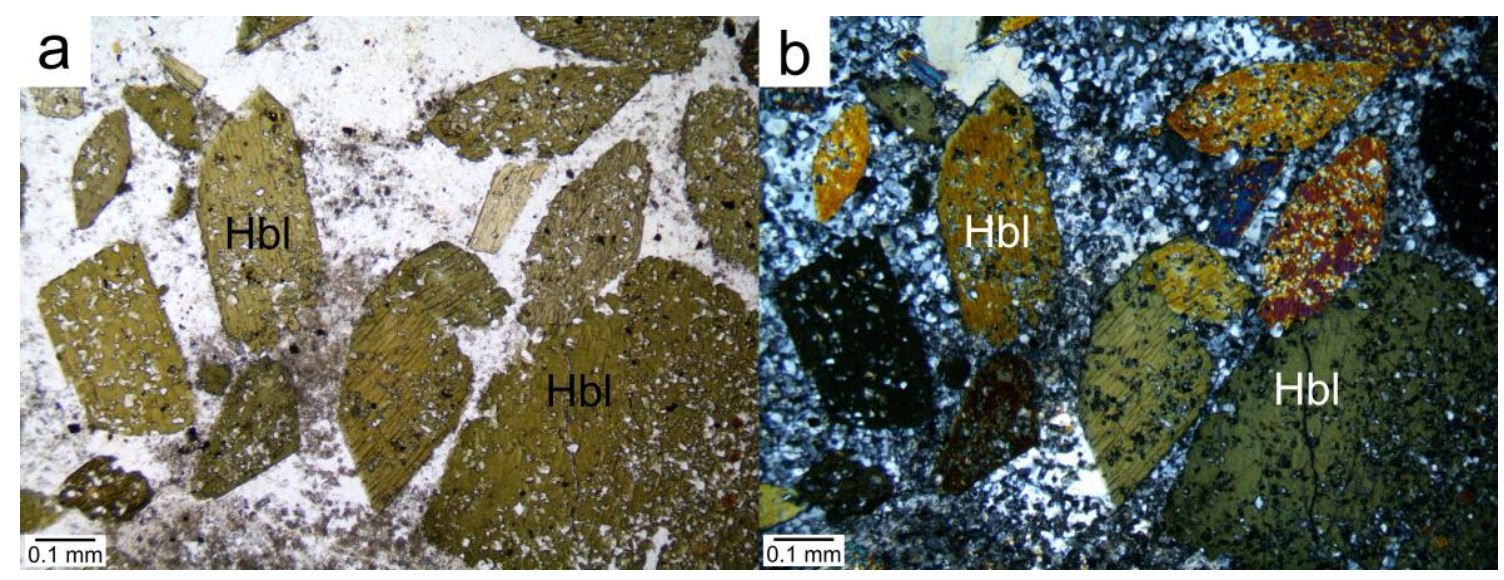

